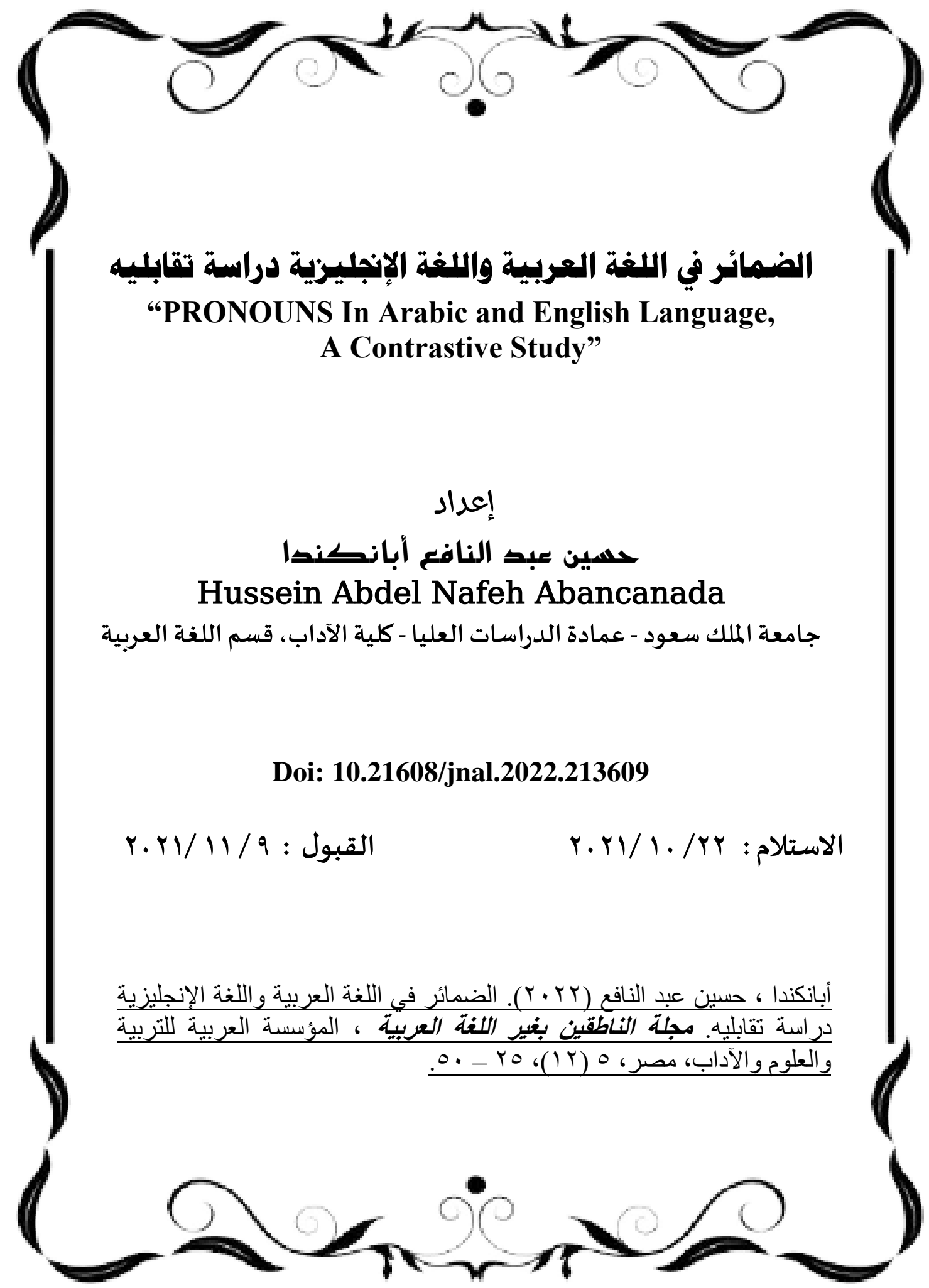




\section{الضمائر في اللغة العربية واللغة الإنجليزية دراسة تقابليه}

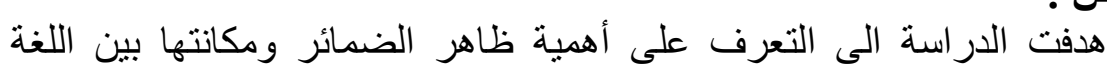

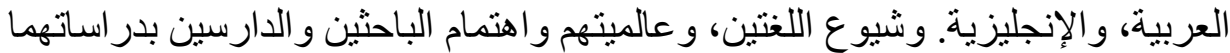

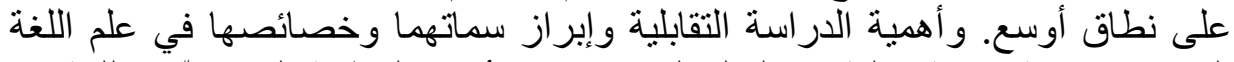

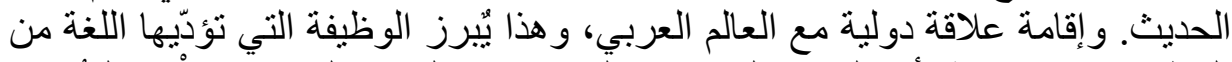

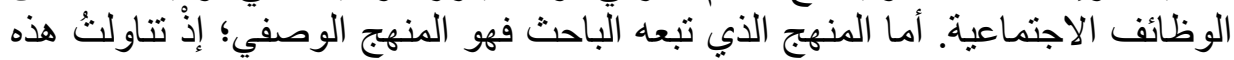

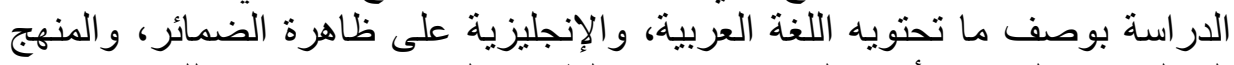

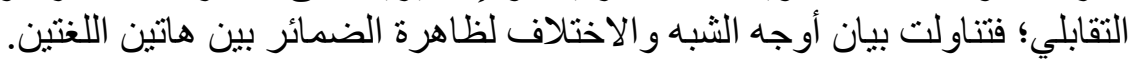

\section{Abstract:}

The study aimed to identify the importance of the apparent pronouns and their place between Arabic and English. The prevalence of the two languages, their universality, and the interest of researchers and scholars in their studies on a larger scale. The importance of contrastive study and highlighting their features and characteristics in modern linguistics. And the establishment of an international relationship with the Arab world, and this highlights the function that language performs among the social functions. The method followed by the researcher is the descriptive method. I dealt with this study by describing what the Arabic and English languages contain on the phenomenon of pronouns, and the contrastive approach; It dealt with a statement of the similarities and differences of the phenomenon of pronouns between these two languages.

مقدّمة:

لا تخلو جملة ـ في أي لغة من لغات العالم- من احتو ائها على الضمير ، وهذ هذا يؤكّد

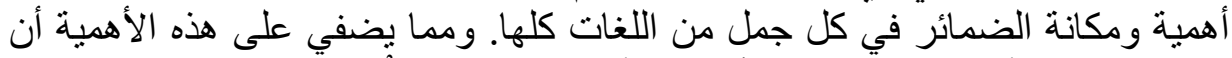

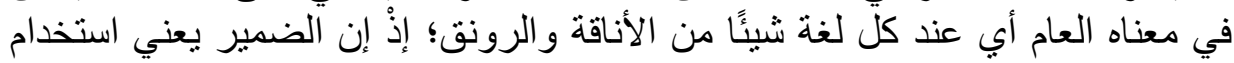

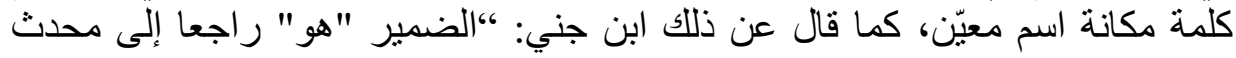


عنه في الكلام السابق "' وفي هذه الحالة فإنّ الاسم المضمر يورث شئيًا من الجمال

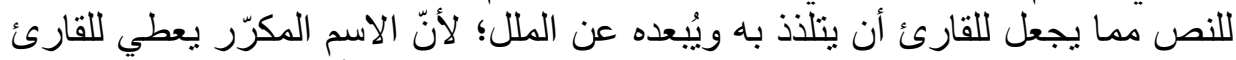

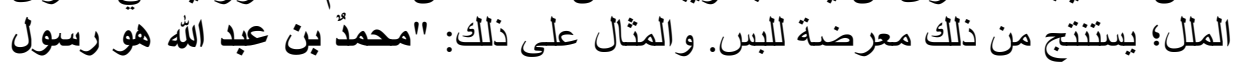

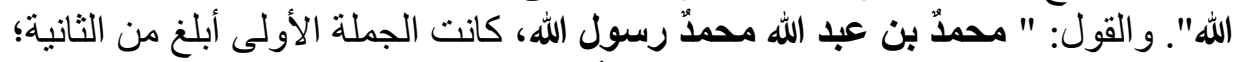

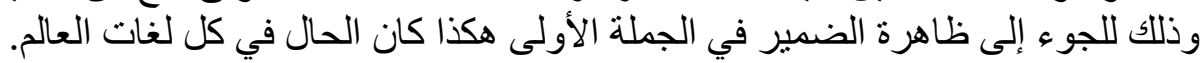

For example, in English language

Muhammad, son of Abdullah, he is prophet of Allah Muhammad, son of Abdullahi, Muhammad is prophet of Allah

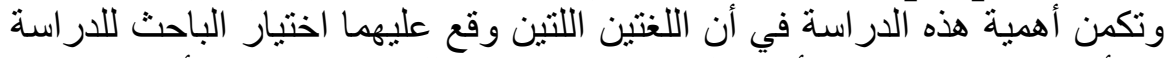

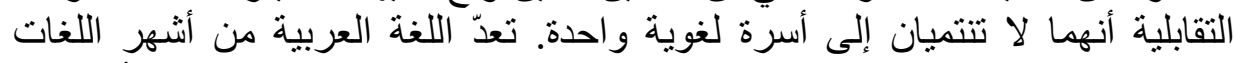

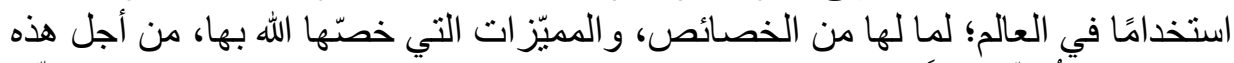

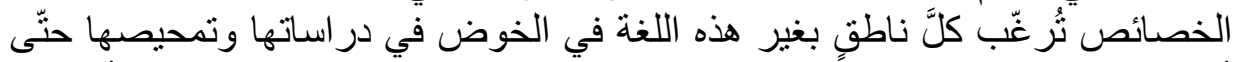

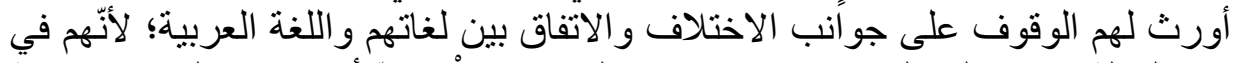

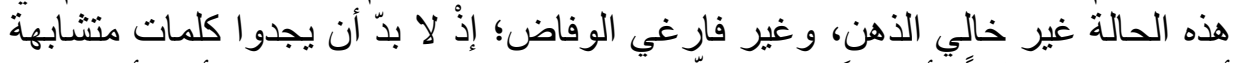

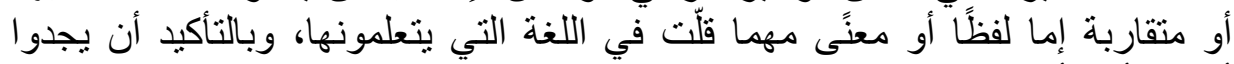

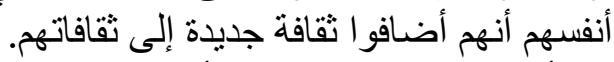

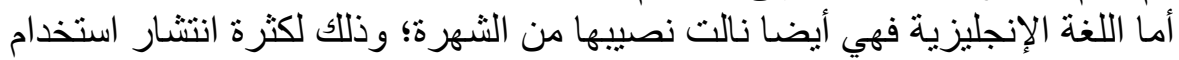

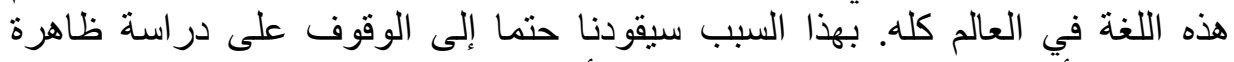

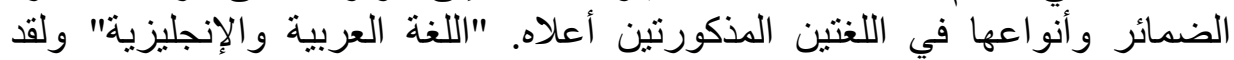
خظيت هذه الدراسة لأى اللسانيين ويطلقون عليها بالدراسة التقابلية ( Contrastive يمكن إدر اك تحديد مشكلة هذه الدراسة بأنّها عبارة عن المحاولة للإجابة عن الأسئلة التالية.

ا ب - مالية. مفهوم الضمائر في اللغة العربية و أنو اعها؟

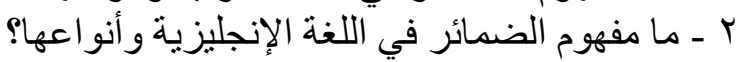

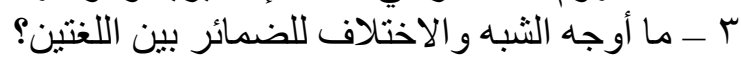

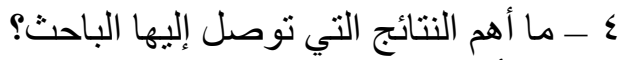

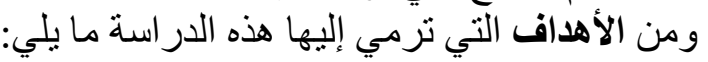
أهمية ظاهر الضمائر ومكانتها بين اللغة العهر التربية، ورالإنجليزية.

' أبو الفتح عثمان بن جني الموصلي، الخصائص، الهيئة المصرية العامة للكتاب، ط/ ع / ج/ TV 
شنيو ع اللغتين، وعالميتهم و اهتمام الباحثين و الدارسين بدر اساتهما على نطاق أوسع.

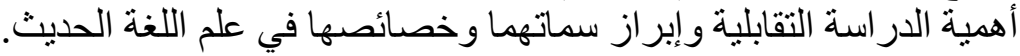
إقامة علاقة دولية مع العالم العربي، وهذا يُّيُرز الوظيفة التي تؤديّيها اللغة من الوظائف

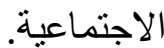

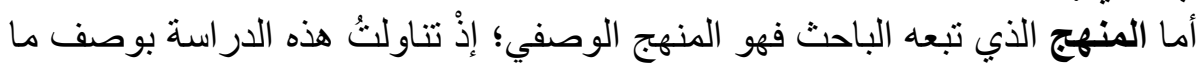

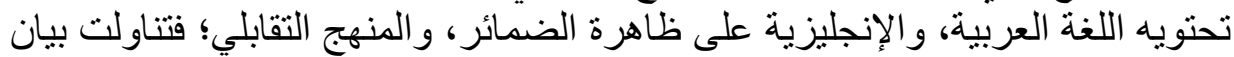
أوجه الثبه والاختلاف لظاهرة الضمائر بين هاتين اللخئ اللغتين.

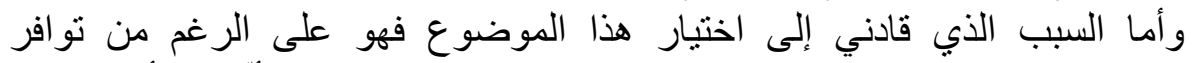

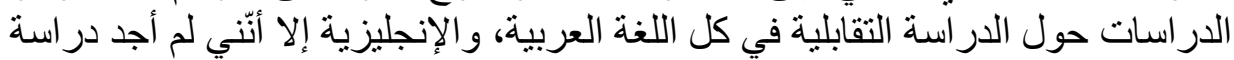
أو بحثا تناول ظاهرة الضمائر. لقد قسمت هذه الدراسة إلى ثلاثة أبو اب، الباب الأول دار على الطى الضمائر في اللغة العربية وشمل ذلك على أنواع الضمائر في هذه اللغة، عود الضمير الضير على الآسم المضمر،

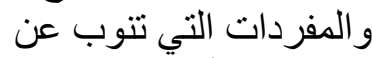

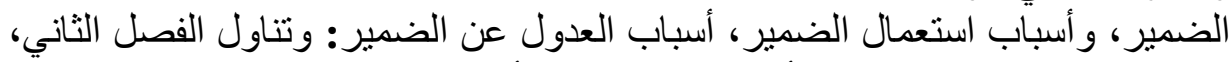

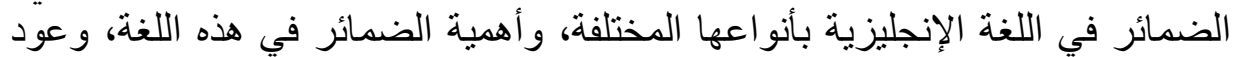

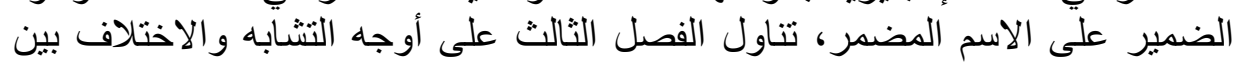
اللغتين، العربية والإنجليزية.

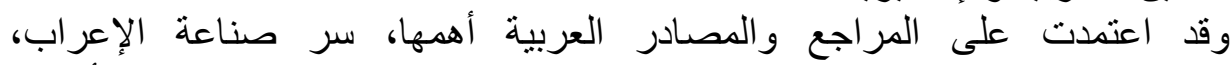

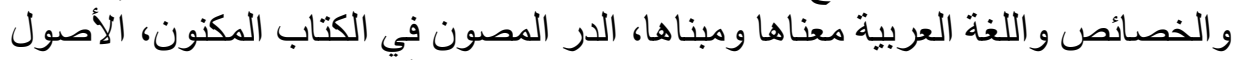

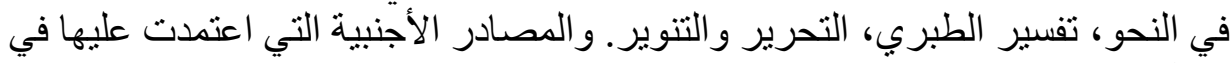

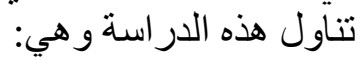

Quick. R and Greenbaum. S University Grammar OF English (long man) London

$\mathrm{J}$. C NESFIED and $\mathrm{F} T \mathrm{~T}$ WOOD, MANUAL OF ENGLISH GRAMMAR and COMPOSITION $4{ }^{\text {TH }}$ EDITION

Gleason H . Linguistics and English Grammar C Holt, Reinhart and Winston New York London

D . N . S . BHAT . PRONOUNS . OXFORD UNIVERSITY PRESS.

Sidney Greenbaum, personal pronouns in present day English, Cambridge universiry 1996.

$$
\text { ولقد توصلت إلى النتائج أهمها. }
$$


و وجود التشابه الدقيق بين اللغتين، (العربية و الإنجليزية). في مفهوم ظاهرة الضمير.

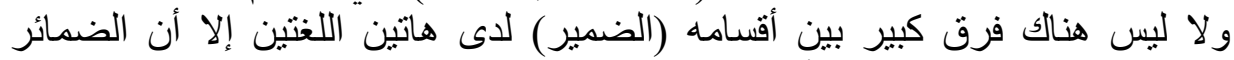

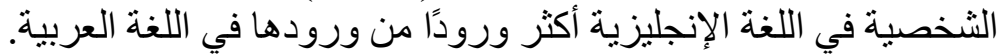
وجود بعض الاختلاف بين اللغتين.

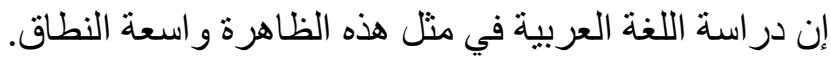

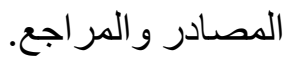

تمهيد:

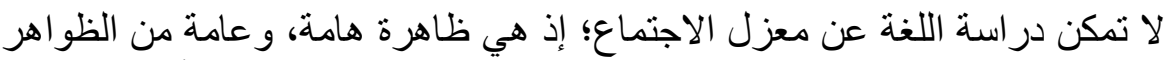

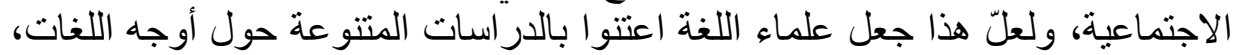

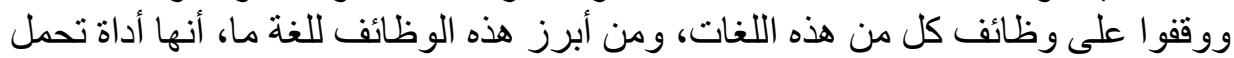

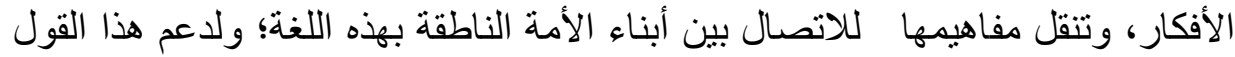

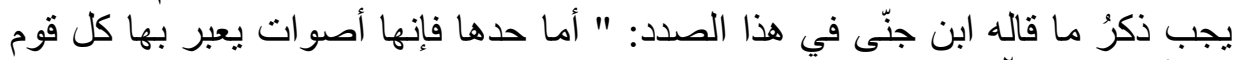

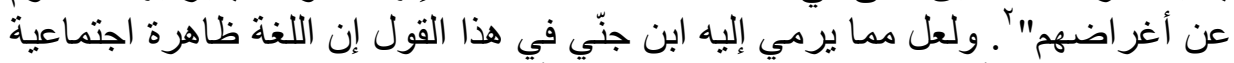

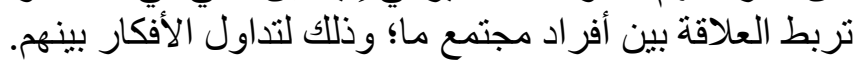

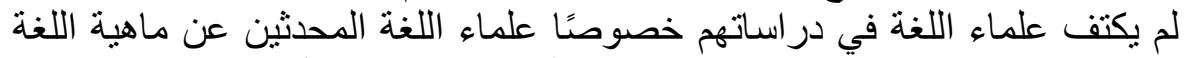

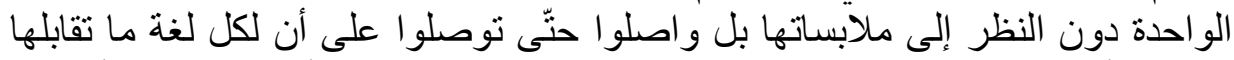

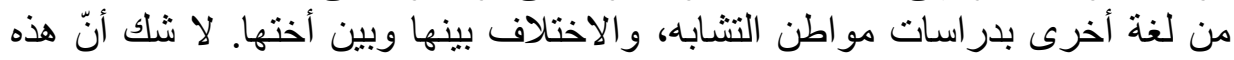

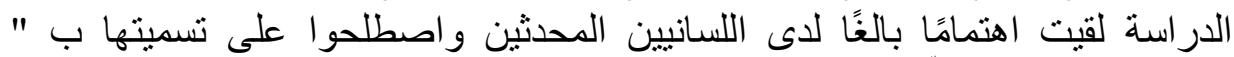

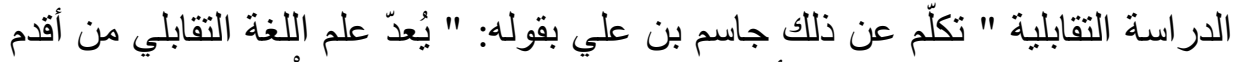

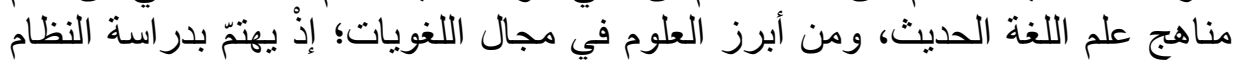

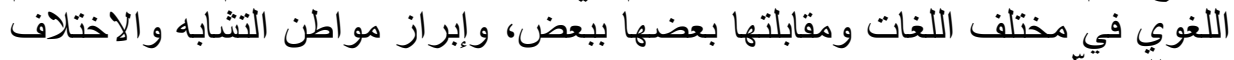

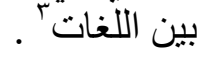

يُفهم من هذا الكلام أن إجر اء المقابلة بدر اسة النظام اللغوي بين اللغات المختلفة؛ بغية

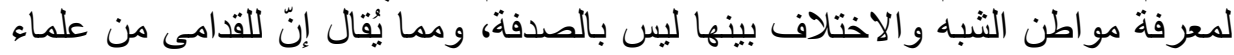

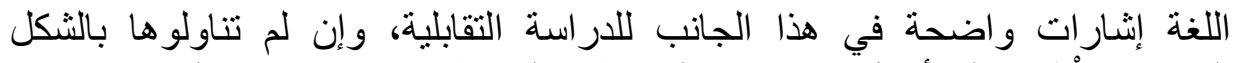

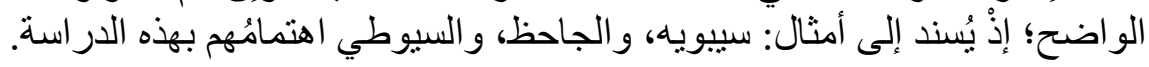

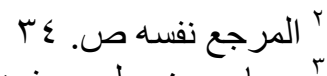

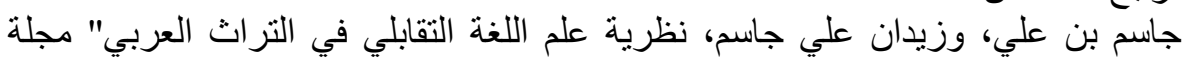

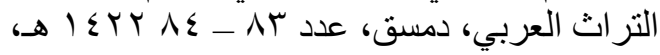

صن 


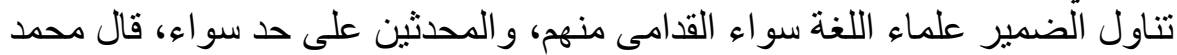

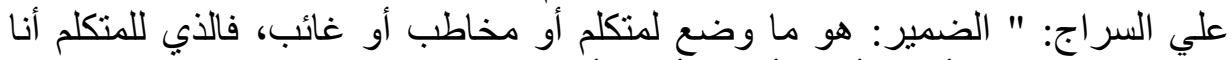

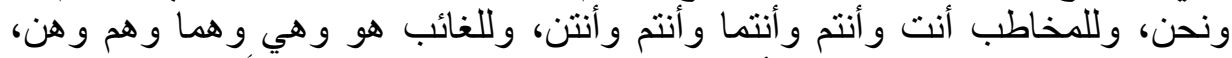

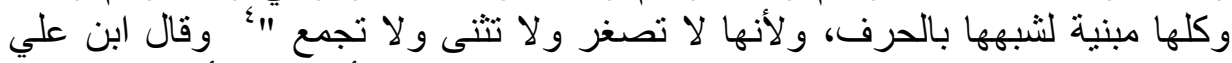

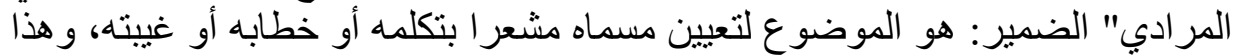

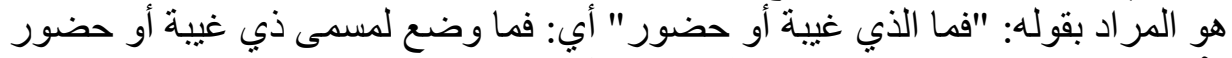

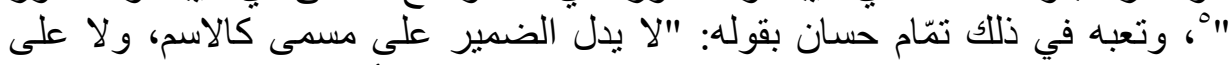

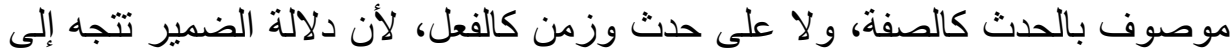

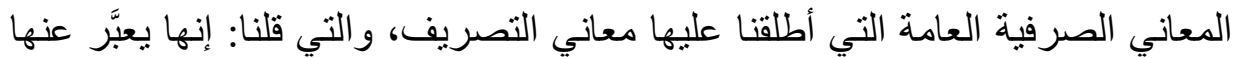

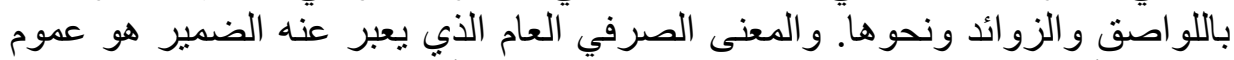

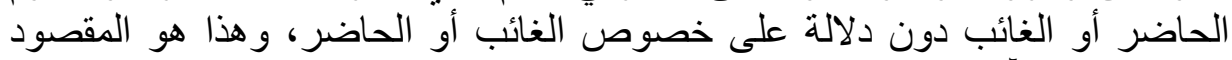

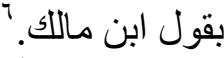
وما لذي غيبة أو حضور ... كأنت وهو سمّ بالضمير.

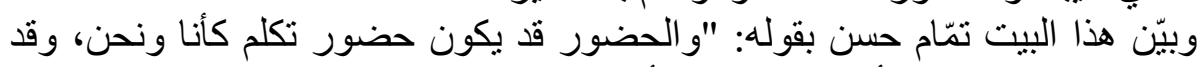

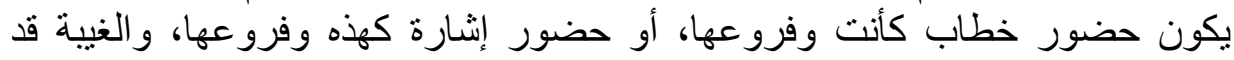

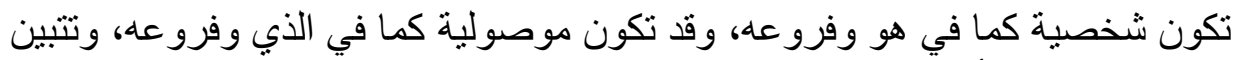

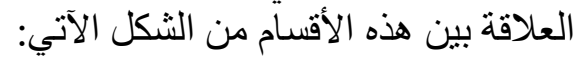
ومعنى ذلك أن الضمائر في اللغة العربية الفصحى تنقسم إلى ثلاثة أقسام، حسب رأى

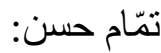

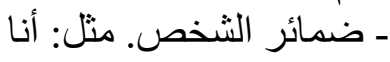

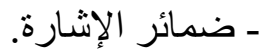

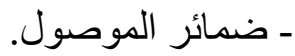

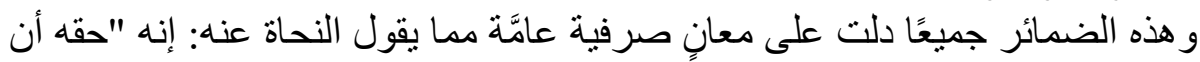

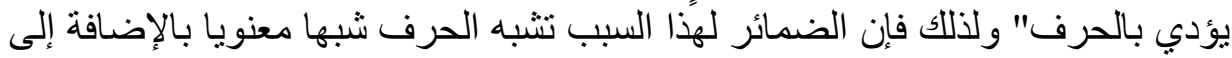
الثبه اللفظي الذي يظهر في بعضها. فلا فارق في الطابع بين معنى الحضور و الغيبة.

" محمد علي السَّراج، اللباب في قواعد اللغة وآلات الأدب النحو والصرف والبلاغة

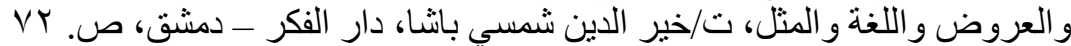

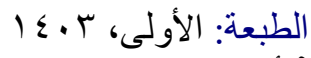
• أبو محمد بدر الدين حسن بن عليَ المر ادي المصري، توضيح الدقاصد و المسالك بشرح ألفية

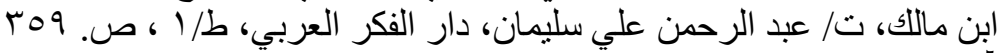

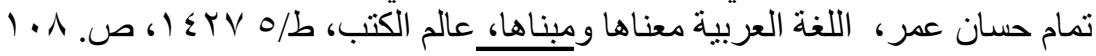


وبين معاني التأكيد و النفي و الاستفهام و الثرط و وابتداء الغاية و المعية و المجاوزة و السببية

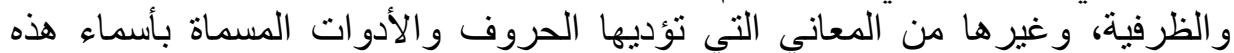

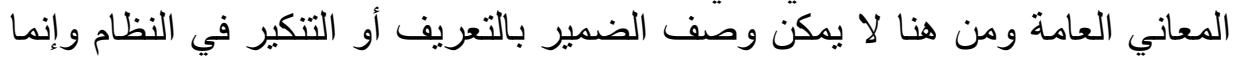

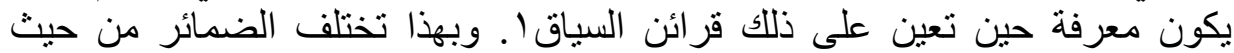

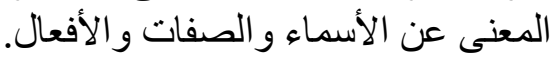

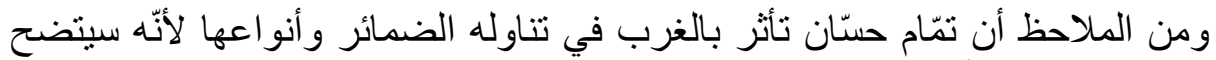

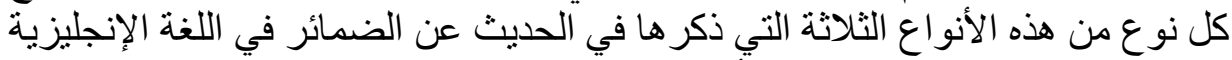

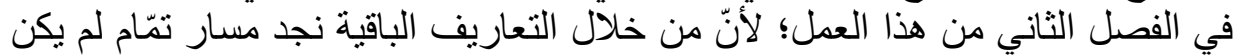

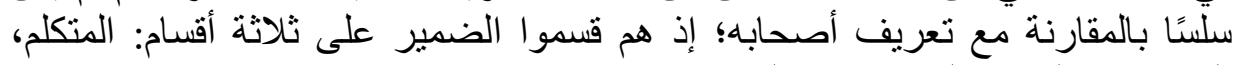

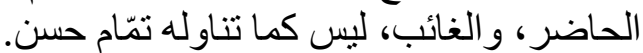

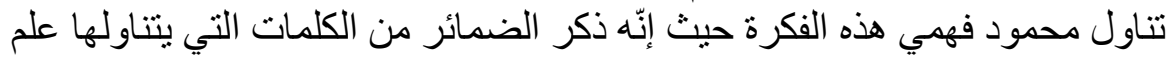

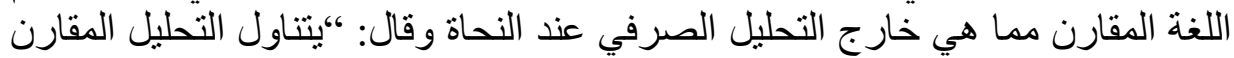

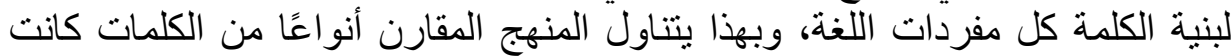

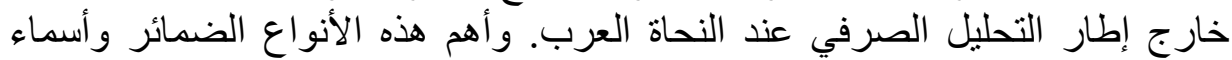

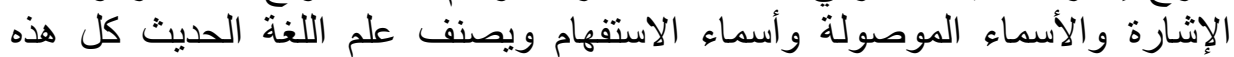

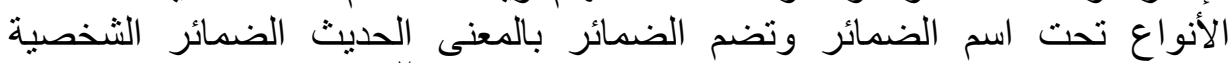

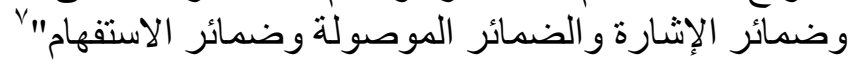
(الضمائر وأنواعها).

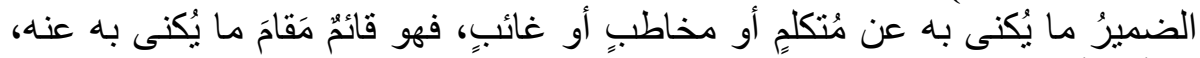

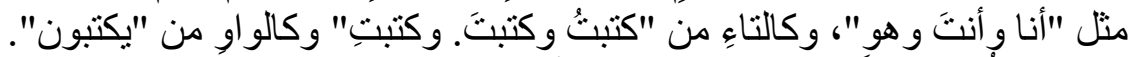

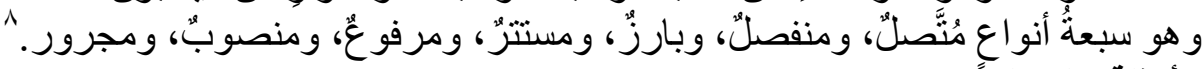
الأمثلة على الضُمِائر.

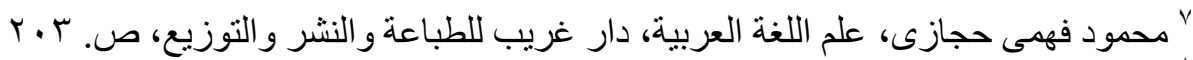

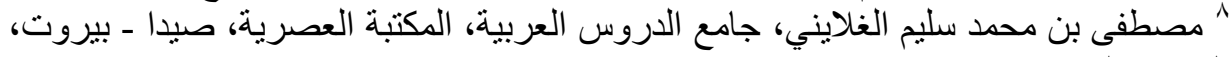

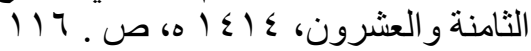




\begin{tabular}{|c|c|c|c|c|c|}
\hline \multirow{2}{*}{\multicolumn{2}{|c|}{ إعداد/محمد حصرية حامد }} & \multicolumn{4}{|c|}{ 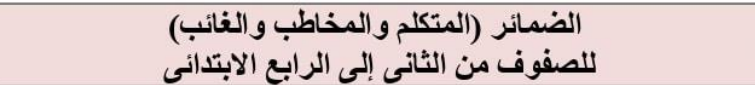 } \\
\hline & & لمخاطب & 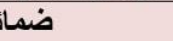 & منكلم & ضمائر \\
\hline هو تلميد & (هو) مفرد مذكر & أنت تلميذ مجنتو & ) & أنا تلميذ & (أنا) للمفرد \\
\hline هل تلميذة & (ه) مفرد مؤنث & أنتِ تلميذة مجتهدة & (َأنتُتِ) للمفرد المؤنتُ & أنا تمبيذة & المذكر و المونتث \\
\hline هما مهندسان & (هما) للمثّى & أنتما مرنذسان مجتهان & (أنتَّا) المثنى & نحن مهندسان & (تحن) للمثنى \\
\hline هما ميندستان & المنكر والمؤنت & أنتتسا مهندستان مجتهدتان & المذكر و المؤثث & نحن مهندستان & المذكز و المؤنت \\
\hline هم أطباء & (هم) للجمع المنكر & أنتم أطباء مجتُهون & (أنتّ) للجمع المذكر & تحن أطباء & (حن) للجمع \\
\hline هن طبيبات & (هن) للجمع المؤنت & أنتن طبيبات مجتهات & (أنتن) للجمع المؤنت & نحن طبيبات & المذكر و المؤنت \\
\hline
\end{tabular}

\section{عود الضمير على الاسم المضمر( سوابق الضمير):}

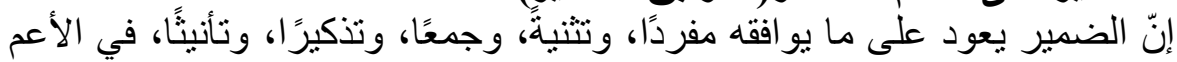

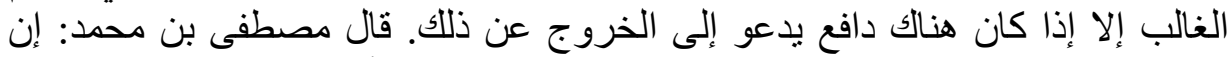

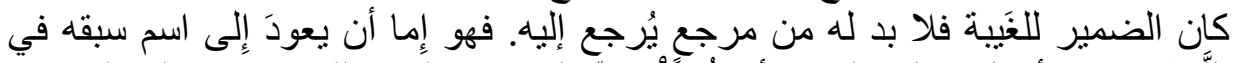

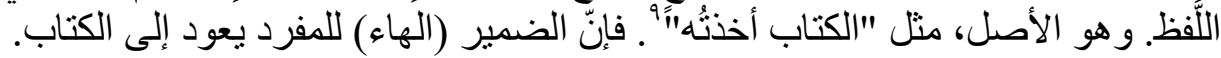

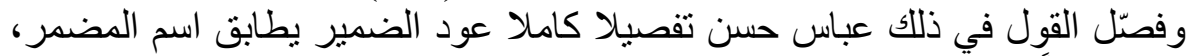

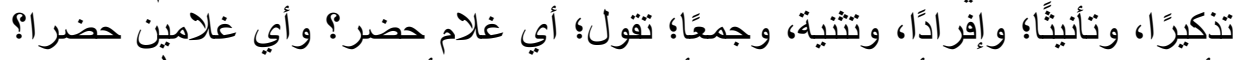

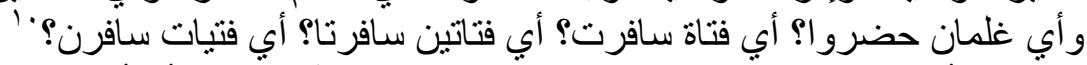

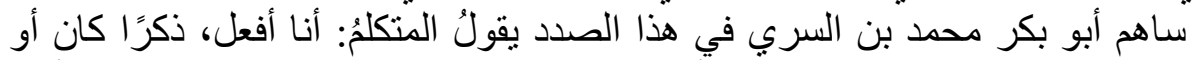

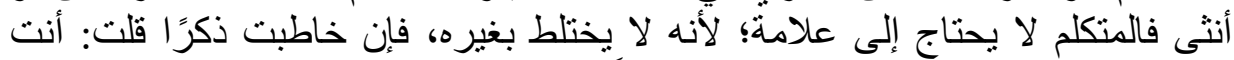

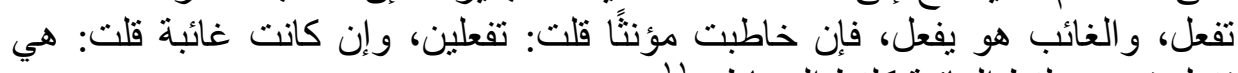

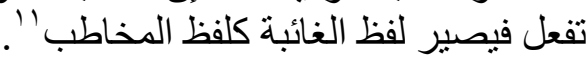

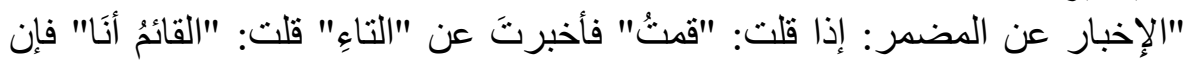

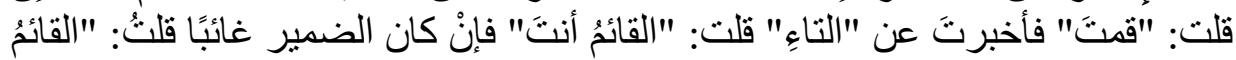

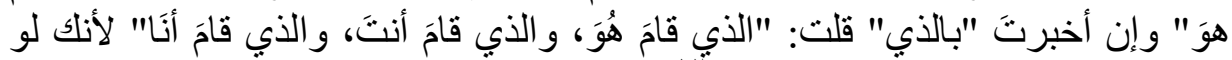

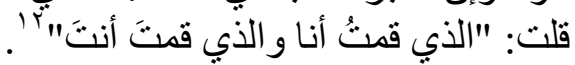

" مصطفى بن محمد سليم الغلايينى، جامع الدروس العربية، المكتبة العصرية، صيدا -

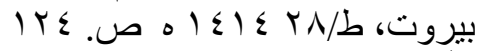

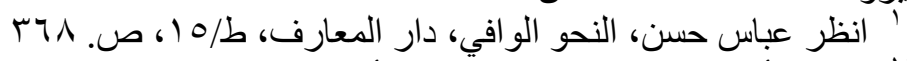

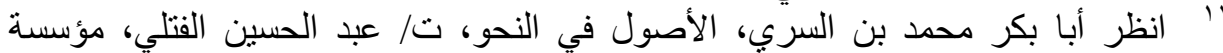

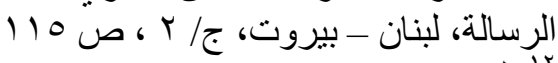

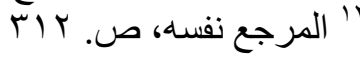


من الواضح اليّيّ أنّ من الأقو ال الواردة أعلاها تضمنت بيانًا عن عود الضمير على

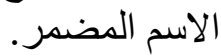
لكن في بعض الأحايين فإنّ عود الضمير ليس على ما يطابقه من اسم المضمر؛ وذلك

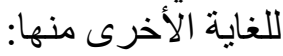

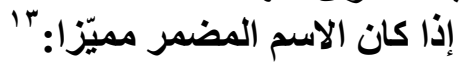

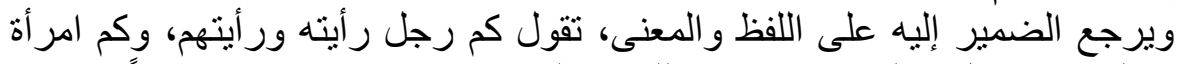

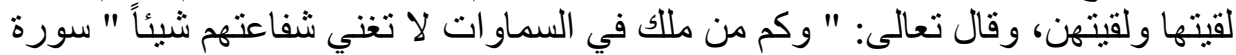

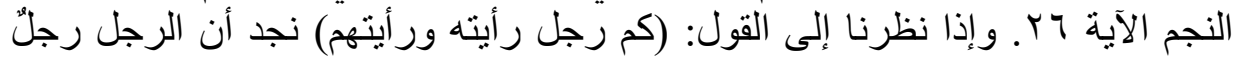

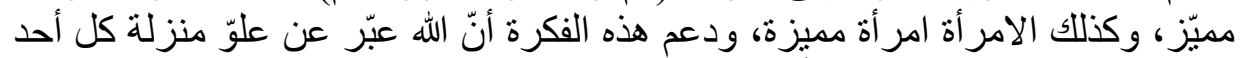

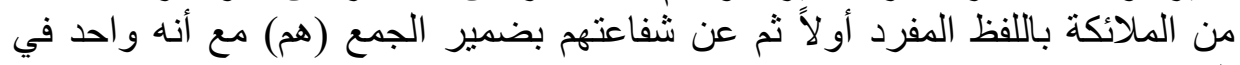

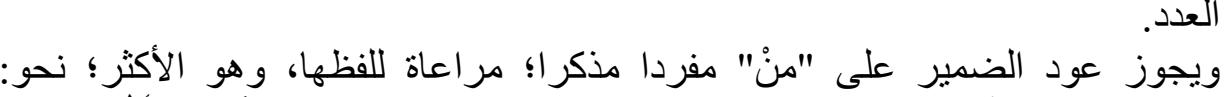

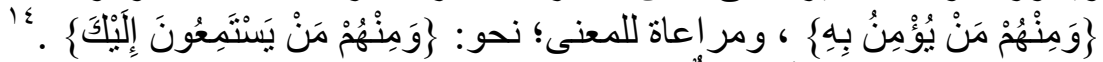

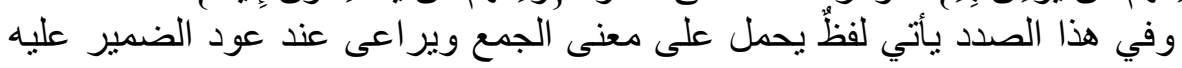

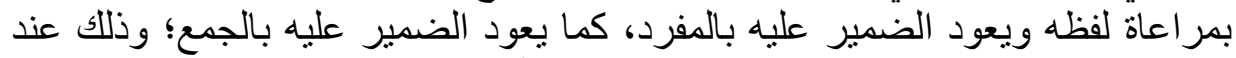
مر اعاة معناه و المثال على ذللك في كلمة (كل): كلّ ينجحُ في امتحانه إلا من لم يُّناكر.

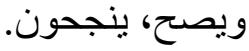
المفردات التي تنوب عن الضمن الضمائر:

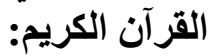
التناوب بين الضمير وبين غيره من الدفردات

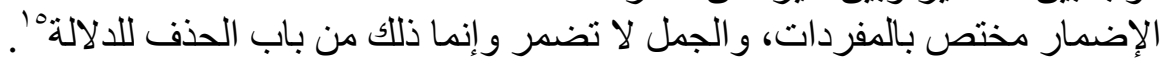

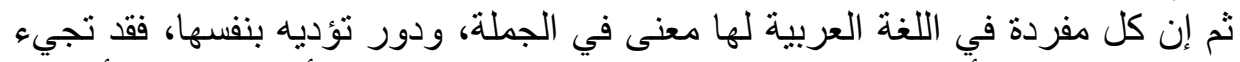

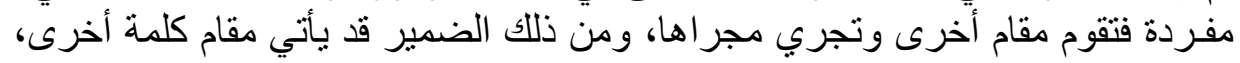

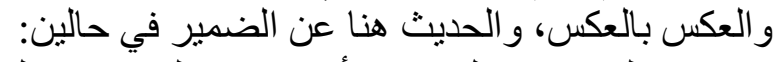
إذا قام الضمير بعمل غيره، أو قام غير الضمير بعمل الضمير الضير.

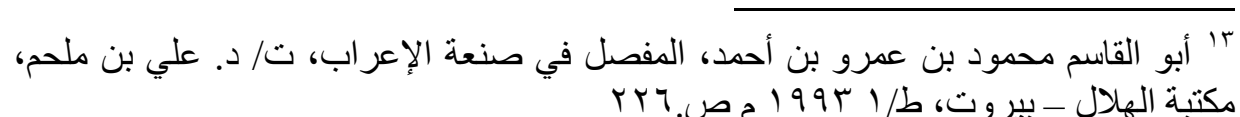

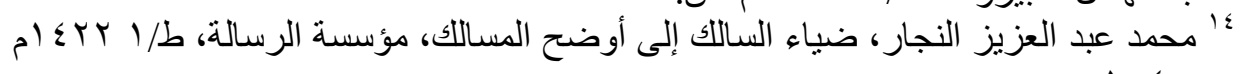
$10 \leqslant .0$

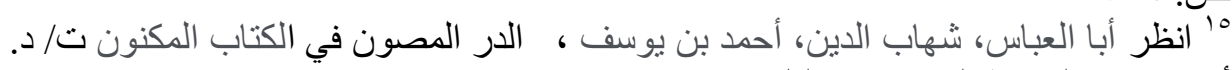

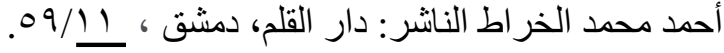
Tr 
النوع الأول: قيام الضمير مقام مفردات أخرى :إن قيام الضرير محل غيره من المفردات

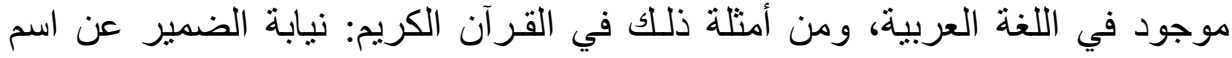

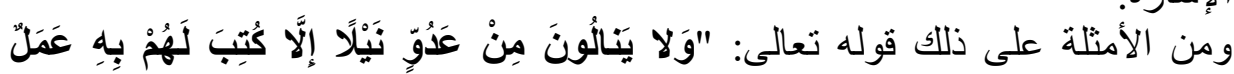

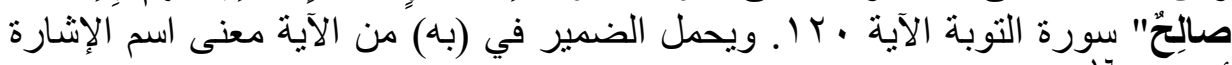
أي بذلك" 17

والذي يظهر من الأمثلة أن اعتبار الضمير بمعنى اسم الإنشارة يكون عندما يعود والئه الضمير على أثنياء سابقة متعددة، والضمير يكون حاله مفرداً، ويحتمل الضمير الضير حينها

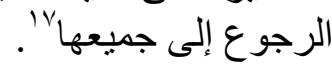

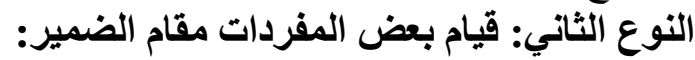

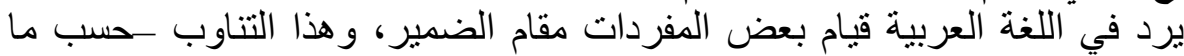

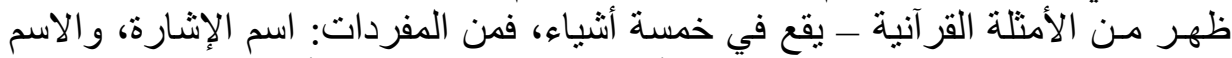

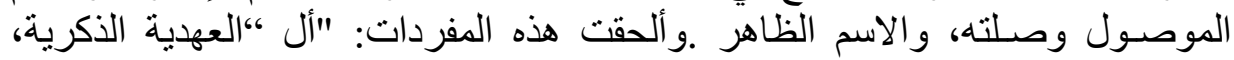

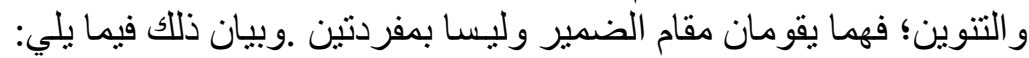

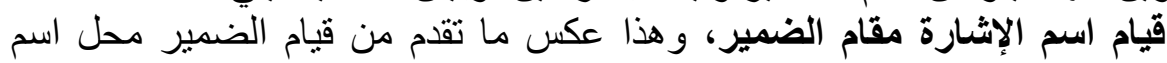

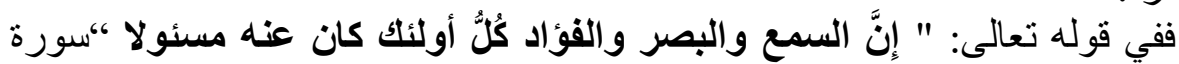

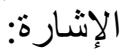

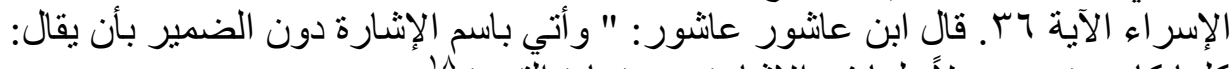

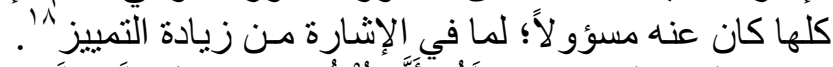

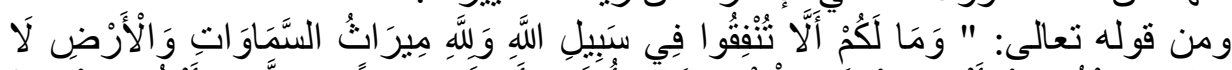

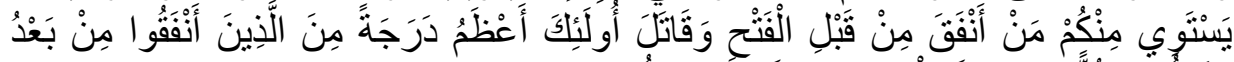

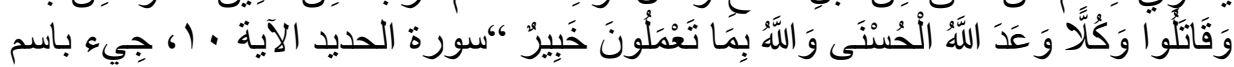
الإشارة بدل الضمير لما يؤذن به الإنشارة من التعظئيم و التنويه.

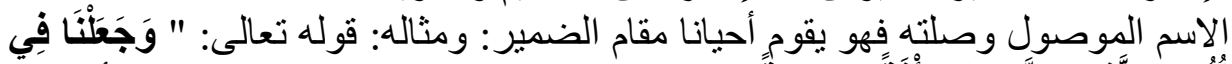

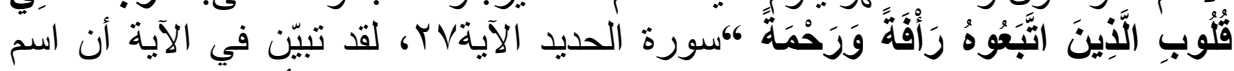

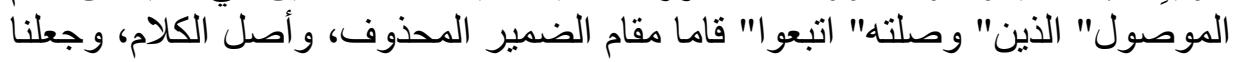

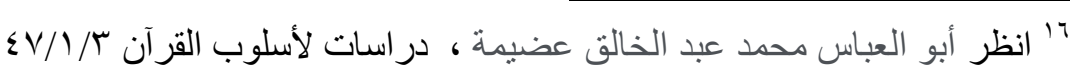

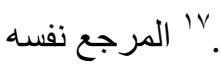

محمد الطاهر بن محمد بن محمد الطاهر بن عاشور التونسي شر النحرير والتتوير،؛ :

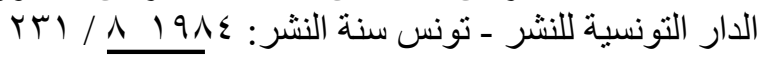
$\Gamma \varepsilon$ 


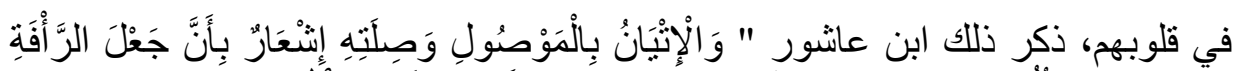

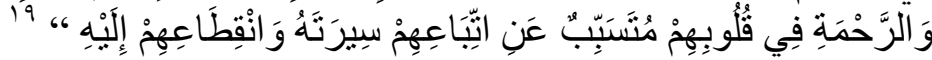

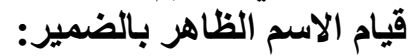
الاسم الظاهر: بأن يكون المقام يقتضي الإضمار فيعدل عنه إلى الاسم الظاهر.

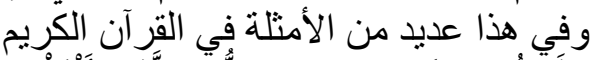

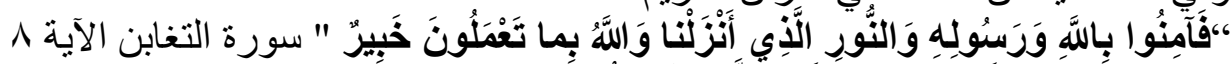

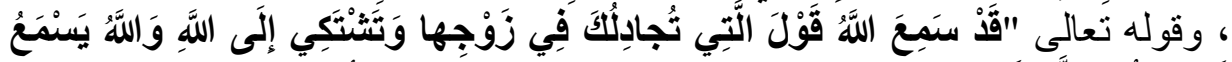

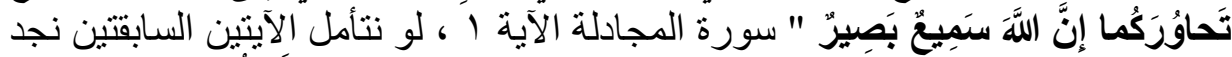

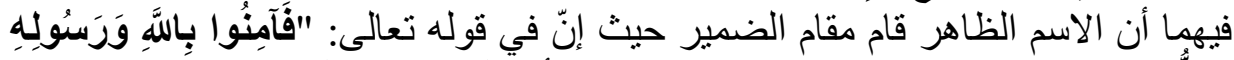

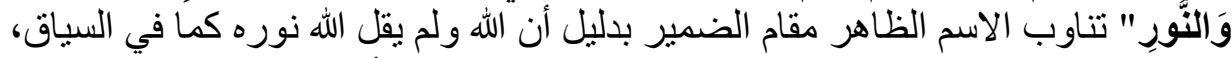

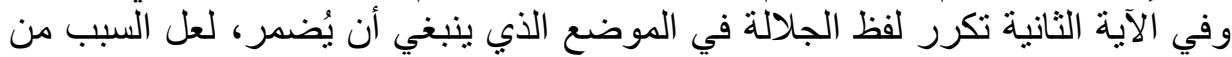

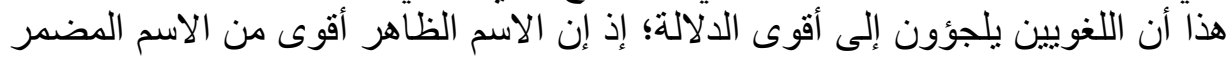

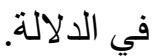
قيام "أل" العهدية الذكرية مقام الضمير: لقد سمى بعض العلماء الضمير : ضمير الذكر

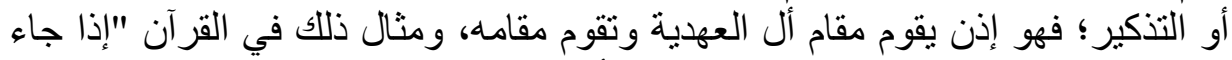

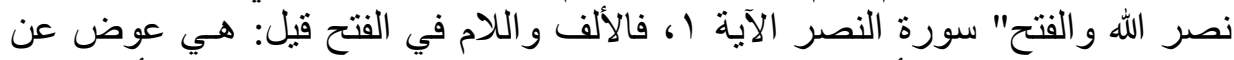
الإضافة عند الكوفيين، أي: وفتحه، و عند البصريين العائد محذوف، وتقديره أي و الفتح

$$
\text { أسباب استعمال الضمير ، وأسباب العدول عنه: }
$$

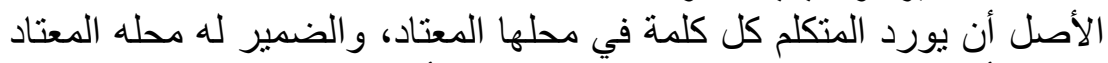

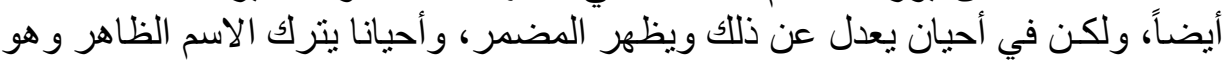

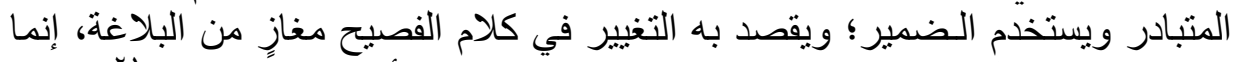

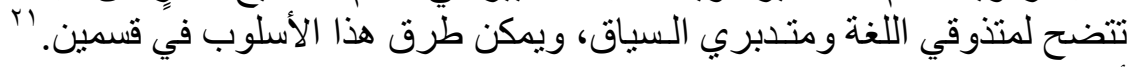

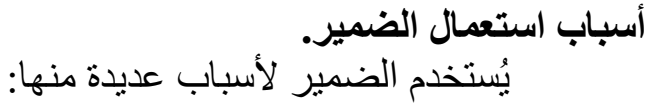

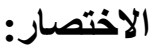

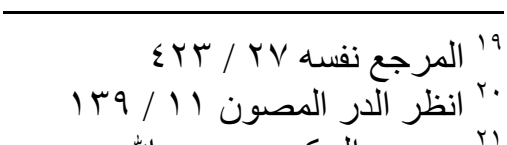

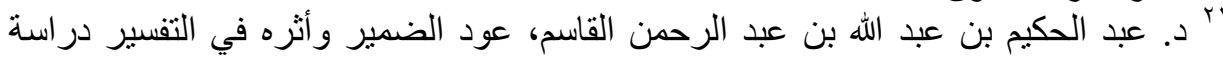

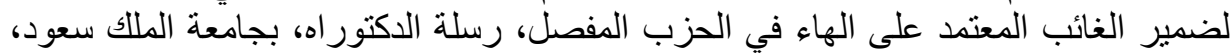

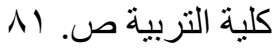




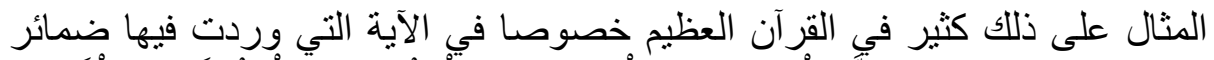

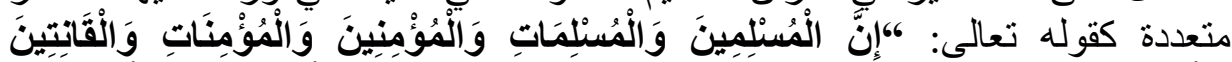

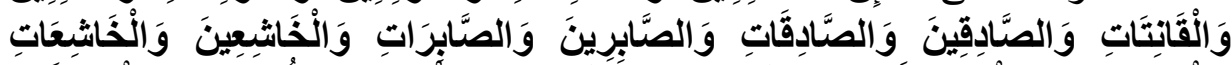

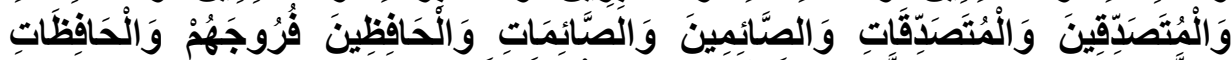

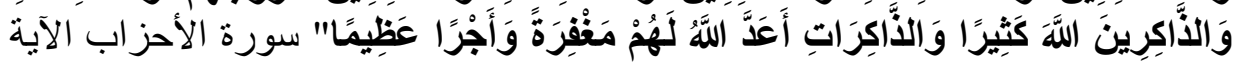

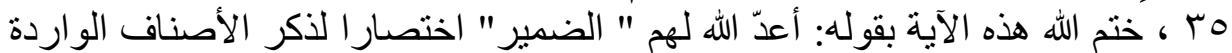

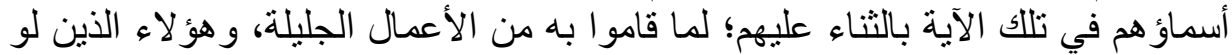

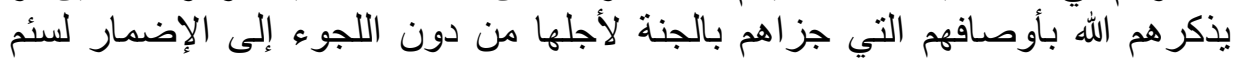
القارئ عن ورود هذه الصفات.

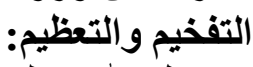

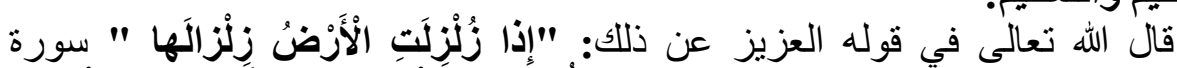

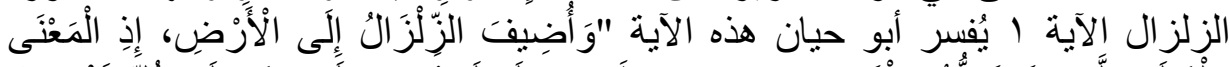

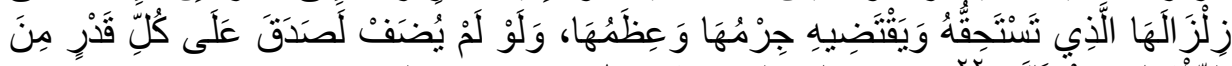

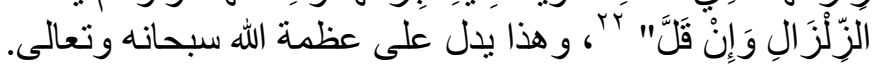

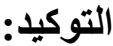

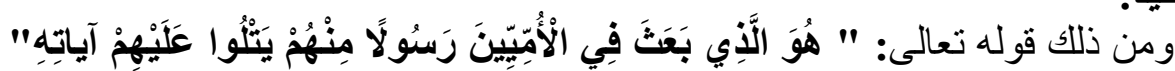

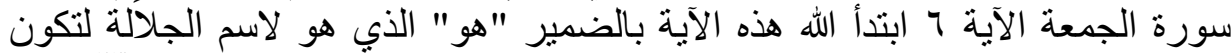

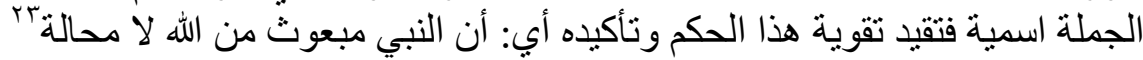

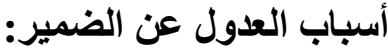
و وهي نوعان، العدول عن الضمير بلا عوض، و والعدول عن الضمير بالعوض.

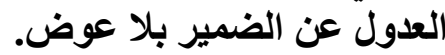
هذه الظاهرة لا تأتي فار غال بل على أسباب عديدة منها: الاختصار بالحذف: الطان

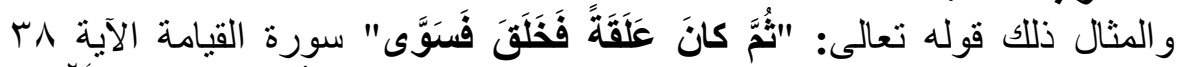

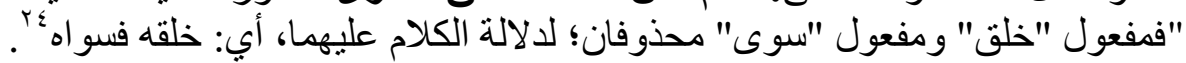

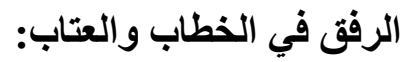

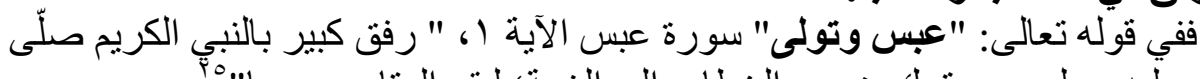

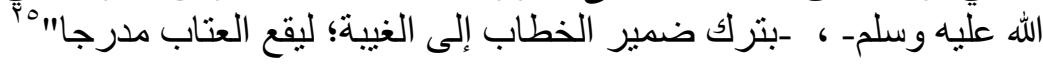

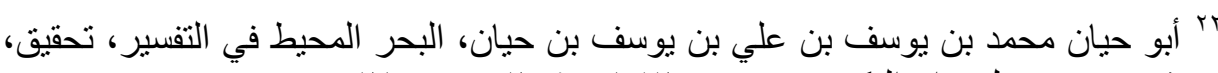

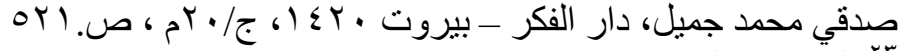

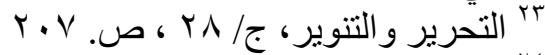

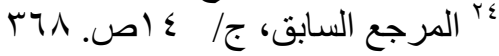


مراعاة رؤوس الآي وسهولة النطق باللفظ:

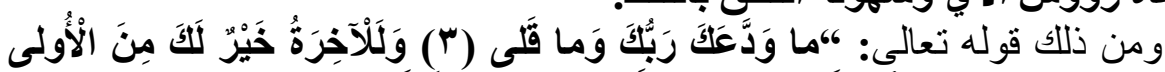

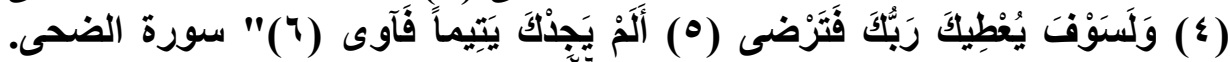

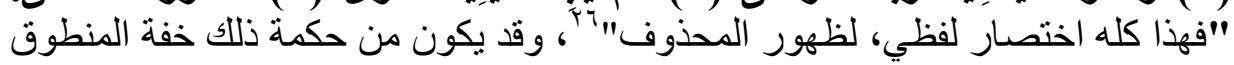

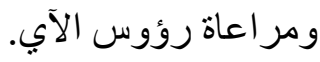

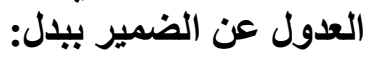

قد يترك الضمير ويُشتبدل مما يقوم مقامه: لأسباب منها:

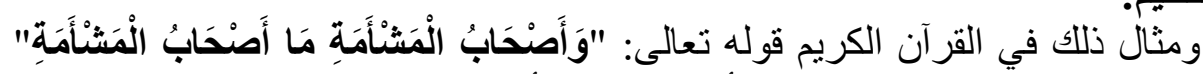

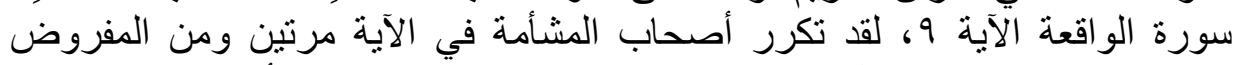

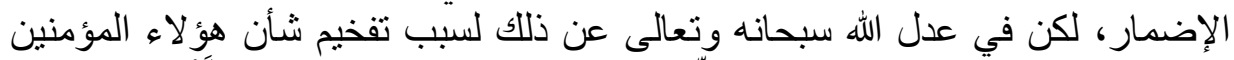

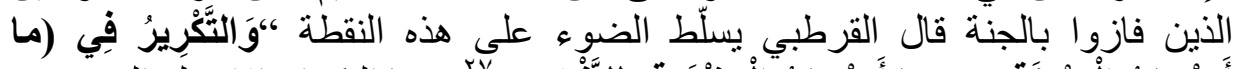

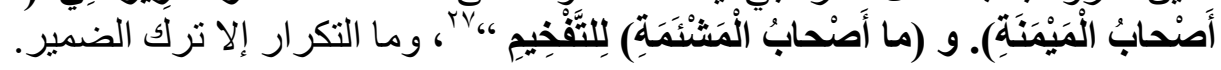

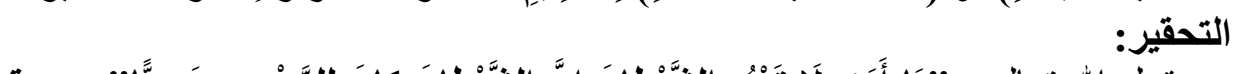

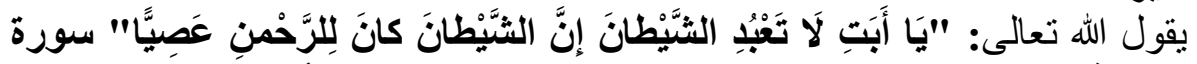

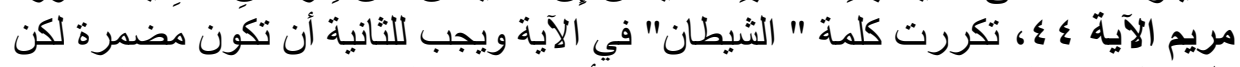

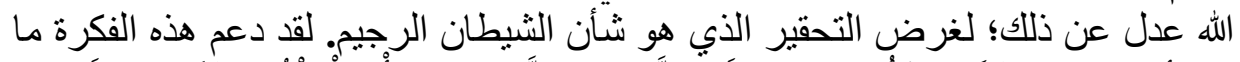

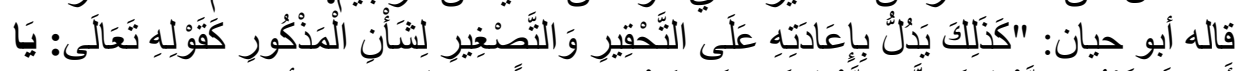

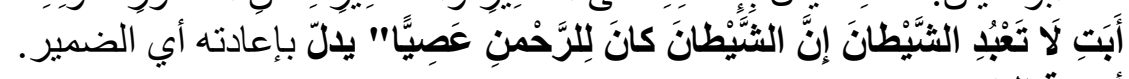

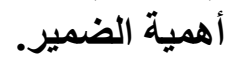
يقول الثناذلي الهيثري: "القول حدث بشري لإنجازه في في جميع اللغات لا بد من:

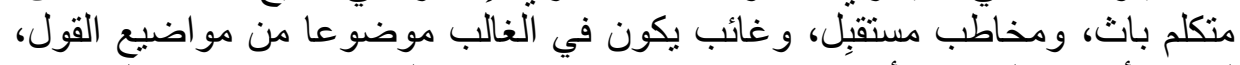

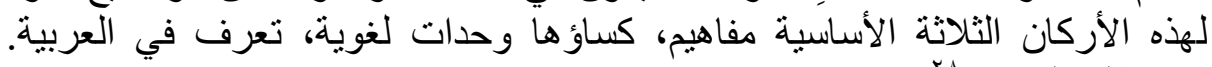

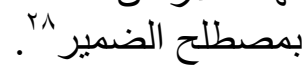

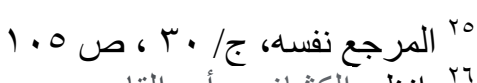

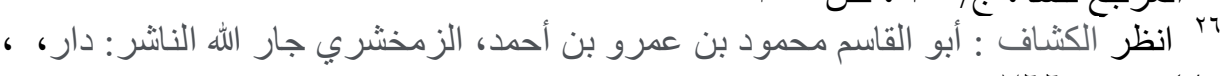

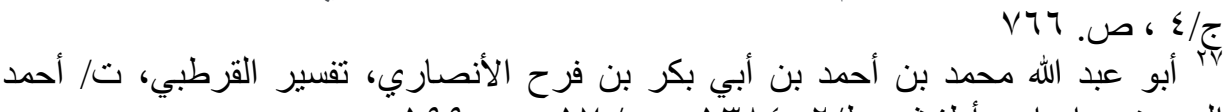

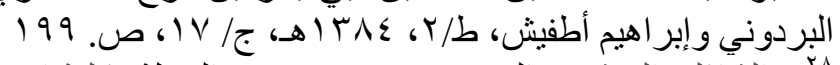

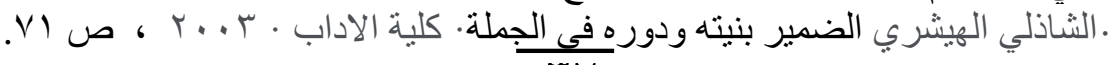
rV 
وقال ابن هثام عن الضمير: " و إنما بدأت به؛ لأنه أعرف الأنواع الستة على لـى

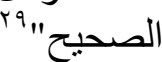

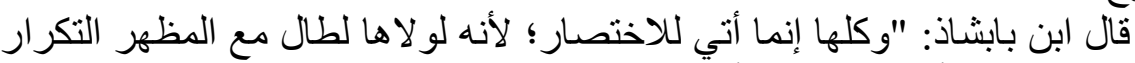

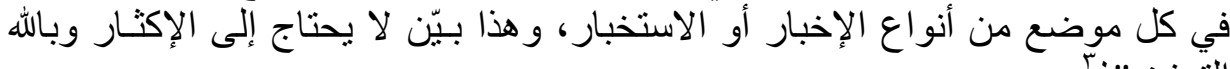
ويُفهم من هذا القول: ما يؤديه الضمير في الجملة من الاختصار الذي يميل إليه

ومما لا شك فيه أن الضمير يلعب دور الربط بين عناصر الجملة قال ابن القيّم:

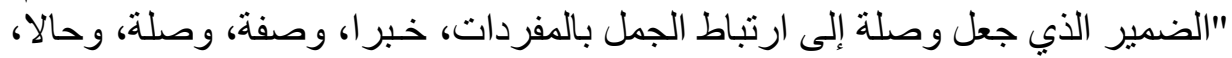

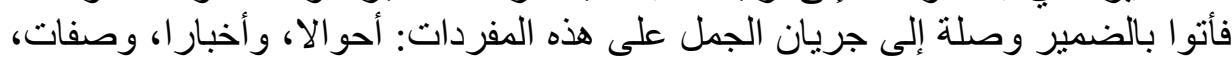

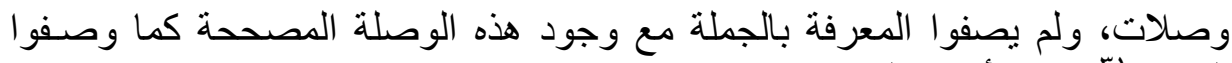

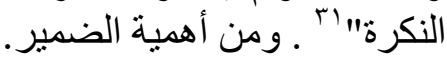
رد الأشياء إلى أصولها: وأمثلة هذه الفائدة أكثر ها صرفية، قال ابن جني: "الإضمار

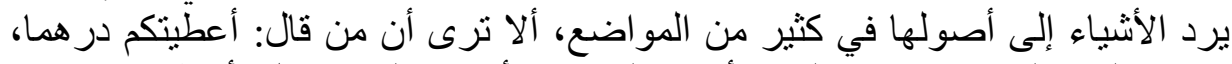

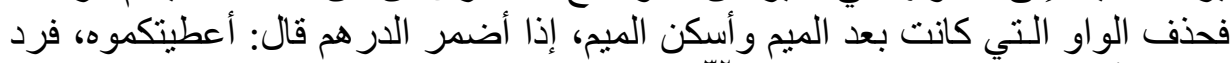

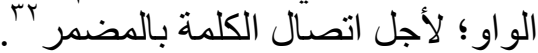

الضمائر في اللغة الإنجليزية: - الية

Pronoun: "Is A Word used instead of A Noun"33 34 The term ’ pronoun' is generally used to referring to several different sets of words such as personal pronouns, demonstratives, e t c " ${ }^{\text {"35 }}$.

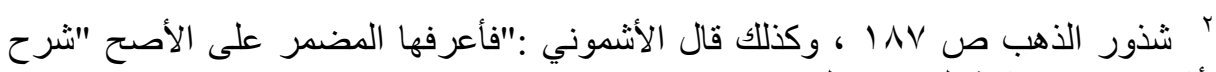

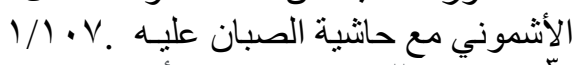
·r مغنتي اللبيب عن كتب الأعاريب عبد الله بن يوسف بن أحمد بن عبد الله ابن يوسف،.

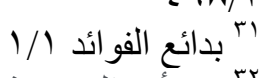

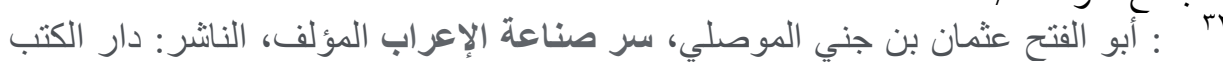

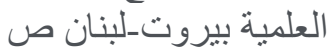

${ }^{33}$ Gleason H . Linguistics and English Grammar C Holt, Reinhart and Winston New York London 1970,p 144-115

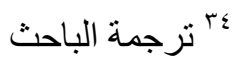


يحمل هذا التعريف على أنّ الضمير يُستخدم عمومًا للإنشارة إلى مجموعات من الكلمات

Pronoun is said to stand for a noun."

$$
\text { مثل: الضمبر الثخصي التعريف. }
$$

$$
\text { "الضمير يقوم مقام الاسم المضمر". }
$$

We understand from these definitions of the pronoun that it represents the nouns that were mentioned previously in the sentence, in order to be short.

إذا نظرنا إلى هذه التعاريف للضمير نجد أنّ كلها تدور في فللك واحد، كما أنّ من الدواعي إلى ذلك أن تكون الجملة قصيرة. Type Of pronouns.

1 - PERSONAL PRONOUNS e .g ; I . YOU, He, She, it.are so called, because they stand for three persons, and have a different form for each ${ }^{37}$

(أ)(الضمائر الشخصية:

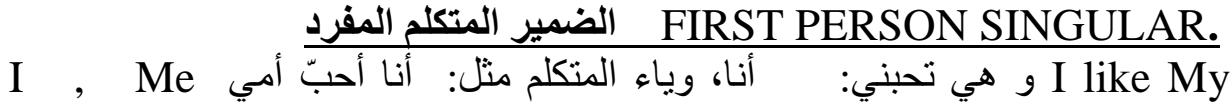

Mummy, and she likes me

الضمير المتكلم للجمع: FIRST PERSON PLURAL

We, us : We give thank to God, he blesses us

الضمير المخاطب المفرد: SECOND PERSON SINGULAR

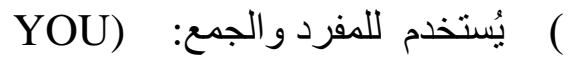

I Like you ( Muhammed) and I Like you(children)

أحبّّك يا محمدًا أحبّكم الأولاد.

الضمير الغائب المفرد. THIRD PERSON SINGULAR

${ }^{35}$ D . N . S . BHAT . PRONOUNS . OXFORD UNIVERSITY PRESS. PAGE 1

36 Sidney Greenbaum, personal pronouns in present day English, Cambridge universiry 1996, page 1

$37 \mathrm{~J}$. C NESFIED and $\mathrm{F} T$ WOOD, MANUAL OF ENGLISH GRAMMAR and COMPOSITION $4^{\mathrm{TH}}$ EDITION, PAGES 40 


\section{مe/ He/ She/it

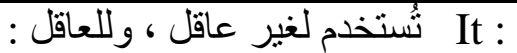

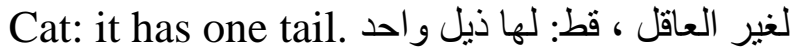
للعاقل: it is you ؟ يخرج هذا الضمير أن معناه الأصلي إلى الاستفهام He; shaeed is a good boy, he is a honest هو : سعيد ولدّر طيّب ، هو صنادق..

: She is a good girl( Zainab) : : Him/ her / it (saheed) I saw him. أ رأيتها الضمير للغائبة : I saw her ( Zainab).her THIRD PERSON PLURAL They / Them

هن / Children : they went out of the class الأولاد: خرجوا / They /

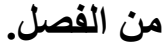
I I saw them / هم ـ Them 2 - REFLEXIBLE PRONOUNS; e .g ; My self, Your self . themselves. These are made by adding "self or own" 38

لا يُستخدم هذا النوع في اللغة العربية بأي نوع من الضمائر بل للتوكيد المعنوي

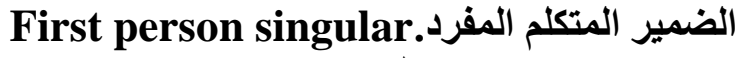

I did it myself فعلته بنفسي

First person plural الضمير للمتكلمين

We did it ourself

Second person singular الضمير المخاطب المفرد

You did it yourself.

Second person plural الضمير للمخاطيين

You did it yourselves يُلاحظ أن هذا يختلف عن الضمير للمخاطبين في القسم الأول من أقسام الضمائر الضمائر الثخصية حيث إن كل you في اللغة الإنجليزية تستخدم للمخاطب ول المخاطبين 3- POSSIVE PRONOUNS ; e.g ; My . Your. Mine. . $^{39}$ 
My hands are warm, but yours are cold

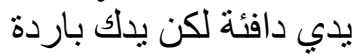
4- INDEFINITE PRONOUS; e. g ; somebody.anybody, anyone, everyone, anything,

(د)ضمائر النكرة :وهي الضمائر الت تستخدم عندما نتكلم عن شخص غير محدد. Somebody told me about that case.

\section{5- DEMONSTRATIVE PRONOUS: $\mathrm{e}$.g this, that. These, ${ }^{40}$}

This pronoun is one that point to some noun or some fact that has already been mentioned.

هذا الضمير هو الذي يثير إلى بعض الأسماء أو بعض الحقائق التي سبق ذكرها.

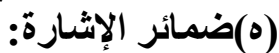

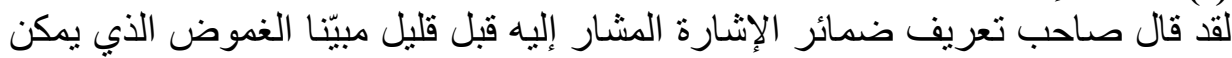

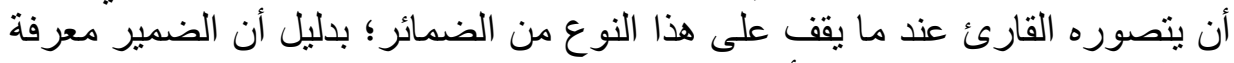

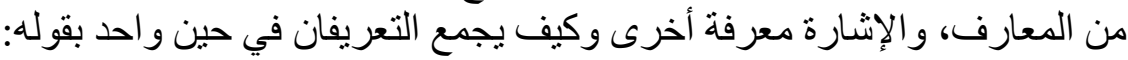
When two nouns have been mentioned in a previous sentences or clause, "this' is a substitute for latter, and 'that' for former; ${ }^{41}$ Example; work and play are both necessary to health, this(play) gives us rest and that (work) gives us energy.

العمل واللعب ضروريان للصحة، هذه (اللعب) تمنحنا الراحة وذاك (العمل) يمنحنا

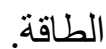

observe that in the sentence " this" does not specify which or what and therefore is not a demonstrative adjective. It is simply put as a substitute for the noun' play" and therefore it is a demonstrative pronoun. ومن الملاحظ أن " هذا" لا يحدد معنى الإشارة في هذه الجملة، و "ذاك" كذلك لا يحدد معنى الإثارة في تلك و إنّما يحمل معنى الضمدير.

${ }^{39}$ John eastwood Ronald, Abasic English GRAMMAR , PAGES 80 40 J.C . Nesfield and F. T. Wood, MANUAL OF ENGLISH GRAMMAR \& COMPOSITION. P .40

${ }^{41}$ Same reference pages 43 
6- RELATIVE PRONOUNS: e $\cdot \mathrm{g}$, which, who, that, not only refers to some noun going before but it also joins two clause together which a demonstrative pronoun does not do ${ }^{42}$ إن أهمية هذا النوع أنه لا يثبر إلى الاسم المذكور مسبقا في الجملة، بل إنّه يربط بين الجملتين معا الذي لا تقوم به ضمانه لائر الإنشارة.

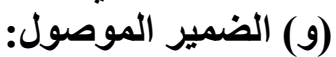

The house in which I live is a good one. المنزل الذي أسكن فيه هو منزل طيب. و هذا الضمير في اللغة العربية يسمى باسم الموصول.

\section{7- INTERROGATIVE PRONOUNS; e g who, which, what. Is one that ask a question ${ }^{43}$}

$$
\text { ضمائر الاستفهام: من أنت؟ Who are you? }
$$

\section{8- Collective Nouns:}

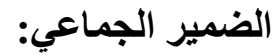

Examples of collective nouns include words such as team, jury, audience,. These collective nouns typically refer to a class or group. Identifying collective nouns as singular or plural can be tricky as the singularity or plurality depends on how the collective noun functions. An antecedent can either refer to the pronoun as a single unit or the different parts of the whole. ${ }^{44}$ بتضح من خلال هذا النوع (الضمير الجماعي) أن الضمير يأني باللفظ، أو الكلمة

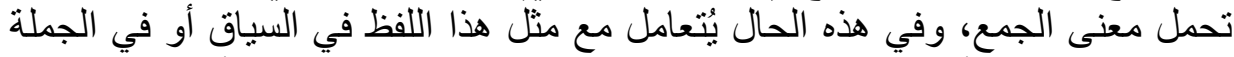
بالنظر إلى لفظه، أو إلى معناه كما هو الحال في اللكمات الواردة في أعلاها. والكال والمثال

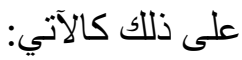

The team won its game, or the team won their game فاز الفريق لعبه، فاز الفريق لعبهم.

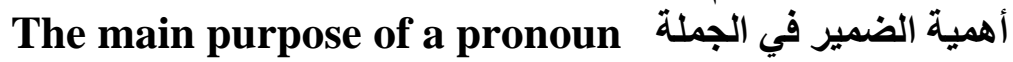

${ }^{42}$ Same reference page 46

${ }^{43}$ Same reference page 48

${ }^{44}$ Written by ryan cheng 
Pronouns are words that replace nouns. People use pronouns to avoid repeating the same noun over and over again (which can become cumbersome). Thus, pronouns allow for a more interesting and concise paper as long as pronouns and antecedents (i.e., the word pronoun refers to) agree in person, number, and gender. ${ }^{45}$

Notice: We benefited from this speech that the pronoun removes boredom from the sentence and bequeaths calls for brevity and economy in the use of paper.

إنّ مما استففنا من استخدام الضمير إز الة الملل في الجملة ويدعو إلى أسلوب الإيجاز

والاقتصاد في استعمال الورقة.

Pronouns are an important part of speech because you use them frequently. And you should use pronouns because they serve important purposes. However, you need to make sure when you use pronouns, you're using them effectively.

Anytime an author uses a first-person pronoun (I, we, etc.) they draw attention to their position and persona. By using "I," a person claims an individual stance, while "we" groups people together. ${ }^{46}$.

يُُستخدم الضمير الثخصي أنا نحن لإبراز شخصية التنكلم وبيان قوة منزلته.

Sometimes pronouns purposefully distance others to construct the speaker's ethos as different. These moves might be politically or racially motivated, as when one group refers to another group of people as "them" or "those people." Here the pronoun itself becomes a type of stereotype. Disidentifying with these others then tells the audience about the values of the speaker. ${ }^{47}$.

${ }^{45}$ D . N . S . BHAT . PRONOUNS pages 15

${ }^{46}$ Bing, Janet. "Killing Us Softly: Ambiguous Markers of Power and Solidarity." Cultural Performances: Proceedings of the Third Berkeley Women and Language Conference. April 1994. 44-49.

${ }^{47}$ Baecker, Diann L. "Uncovering the Rhetoric of the Syllabus: The Case of the Missing I." College Teaching 46.2 (1998): 58-62. 
نفهم من هذا القول إنّ الضمير في الأصل معرفة وقد يخرج عن هذا الأصل (المعرفة) إلى النكرة كما اتضح ذلك في المثال السابق. هؤلاء الناس عندما نعبّر عن الضمير them. للغائين النكرة النين

We is a rhetorical device that allows the speaker(s) to distance themselves from whatever is being said, thus making it appear more palatable because it appears to come from the group as a whole rather than a particular individual" "A يُّنير الضمير (نحن) إلى أداة بلاغية تتيح (المتحدثين) إبعاد أنفسهم عما يقال، مما يجعله

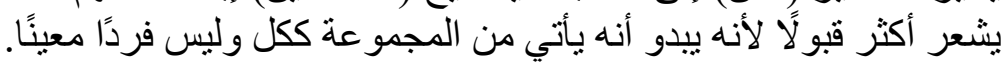

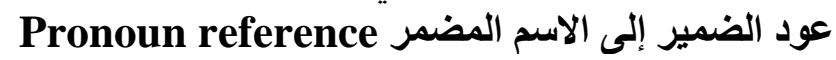

The main purpose of a pronoun is "to replace" a noun. The noun a pronoun replaces is called an antecedent. Pronouns, though, need to be coordinated with their antecedents. If they're not, confusion quickly emerges for readers.

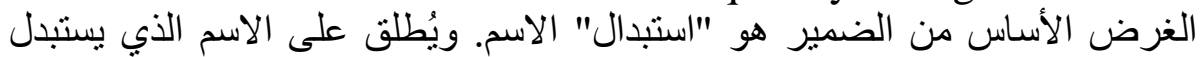

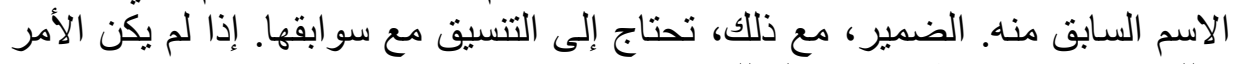
كذلك، فسر عان ما يظهر الارنباك للقر اء. Pronouns should agree with their antecedents in number, gender, and person. For example, if the antecedent is a singular noun, the pronoun should be singular. If the noun is a gendered noun referring to females, the pronoun also should be gendered appropriately. If a sentence is written in the second person, it should remain in that person and not keep switching between first, ". .second, and third person. Below are some examples يجب أن تتفق الضمائر مع سو ابقها في العدد و الجنس و الثخص. على على سبيل المثال،

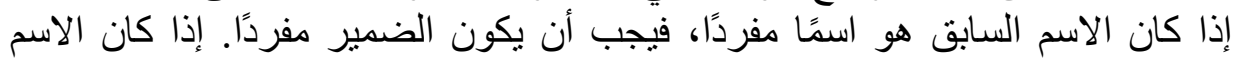

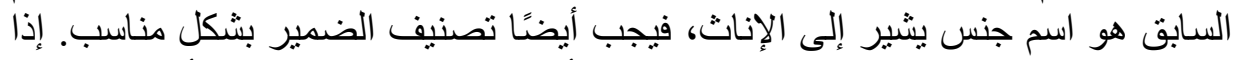

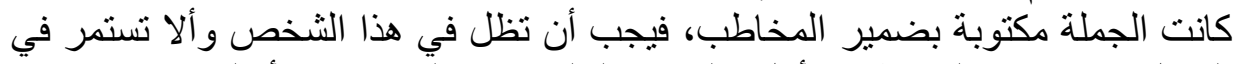
التبديل بين ضمير المخاطب الأول و الثاني و الثالث. فيما يلي بعض لنال الأمتلة.

${ }^{48}$ Same reference

${ }^{49}$ Joseph M moxley 
The boys walked across the street, they ran across the field

Notice: in this sentence "the boys" is plural and in the third person, the appropriate pronoun for it is they which also in the third person and plural.

In modern hospital, a good doctor should always be pleasant to his or her patients.

Notice: in this sentence doctor is a singular and third person, the appropriate pronoun for it is his/her which is third person and singular.

إنّ الدراسة التشابه والاختلاف للضمائلة في اللغة العربية والإنجليزية. المختلفتين، أو اللغات المختلفة، وإنّ أوجه التشابه والته والاختلاف في استعمال الضمائر

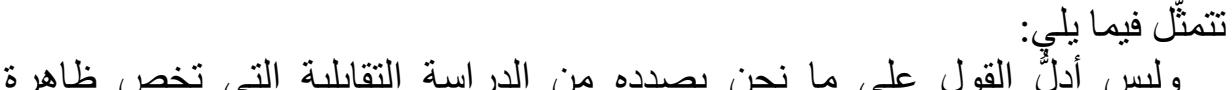

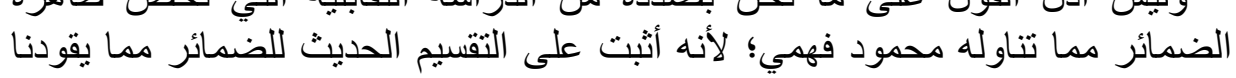

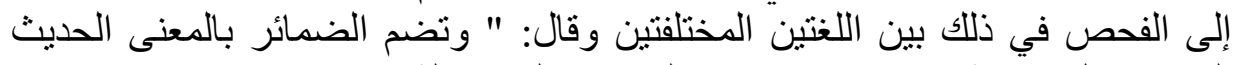

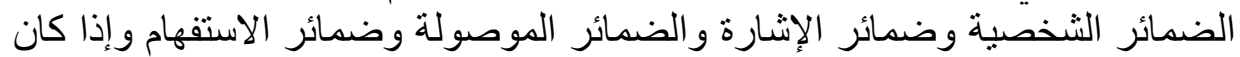

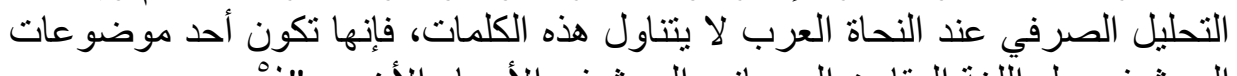

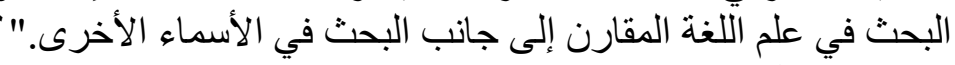

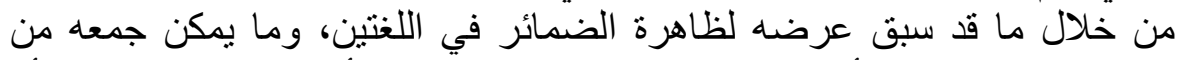

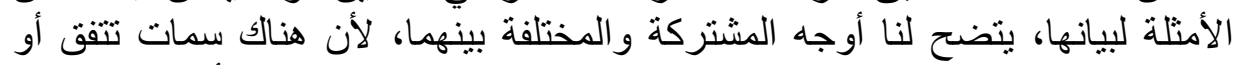

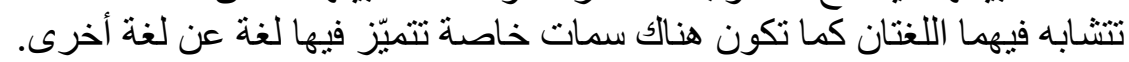
الملامح المشتركة أو الأوجه المتفقة في اللغتين.

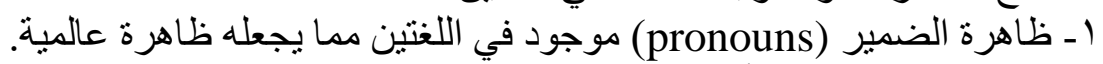

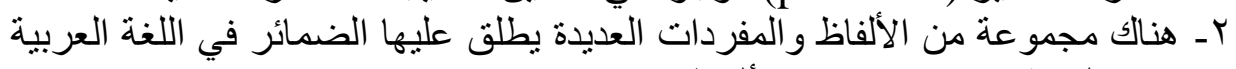
وفي الإنجليزية وبينهما تشنابه في ألفاظهي الإنها.

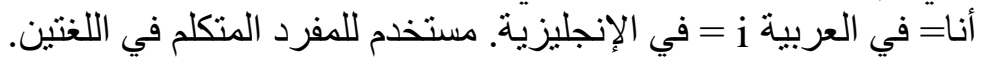
هو= في العربية he = في الإنجليزية. مستخدم للمفرد المتكلم في اللغتين. نحن= في العربية we = في الإنجليزية. مستخدم للمتكلمين في اللغتين.

• محمود فهمي، علم اللغة العربية، ص. ب. ب 
r ال - وقوع الضمير في جملة أساسية وهذا يدلّ على تعبير، وتركيب أساس في اللغتين وليس المقصور على التوضيح و التيبيان فقط. ع - وظيفة الضمير في اللغتين الإيجاز والاختصار في القول كما سبق عرض ذلك في الفصل الأول و الثاني، وبيان ذات سابقة.

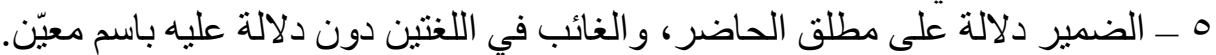

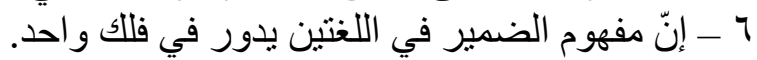

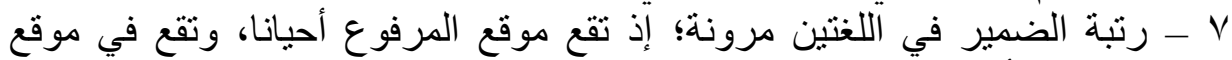

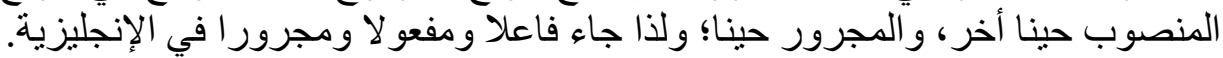
1 - تعتبر هذه الظاهرة (ضمير) من المعارف في اللغتين و لا يحتاج إلى إضافة المعرفة الإلية الأخرى إليه عند اللغتين. 9 - يمكن الاجتماع بين لفظ الضمير و الإثارة في اللغتين ويتضح ذللك فيما قاله تمام

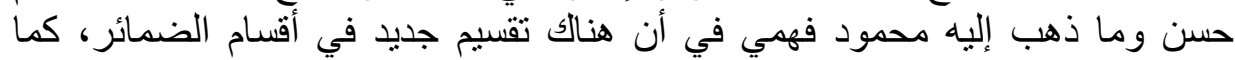

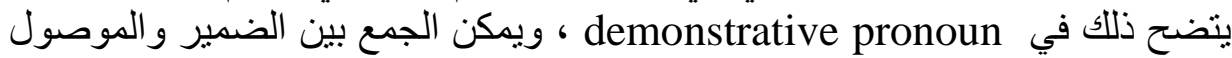

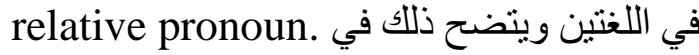
10 - هناك حرية تامة للضمير في اللغتين؛ إذ تقع تارة صدر الجملة، أو في وسطها أو في طرفها. 11 - يدل الضمير في بعض الاحايين على إبراز شخصية المتكلم كما يدل على في

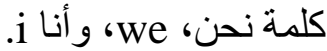
r العالك، - هناك عدد من الضمير يستخدم محايدة في اللغتين: هاء: يستخدم للعاقلة وغير

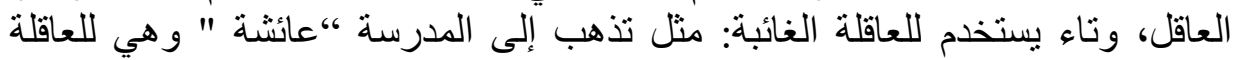

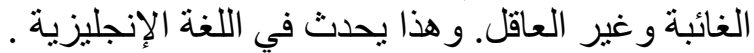

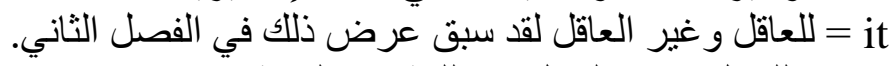
has = للاقاقل وغير العاقل في اللغة الإنجليزية. أوجه الاخلاف للضمير في اللغتين:

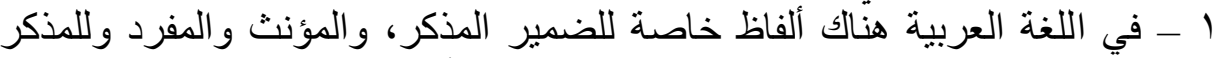

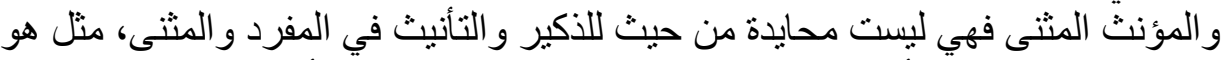

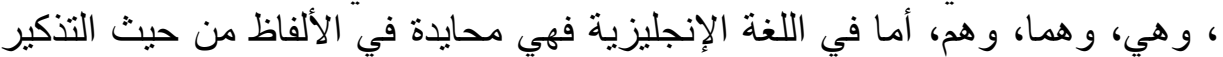

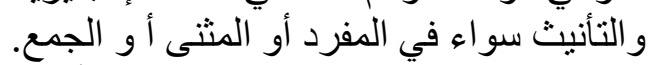
r - بشترط في اللغة العربية مطابقة ألفاظ سوابق الضئ الضمير من حيث العدد والإفراد

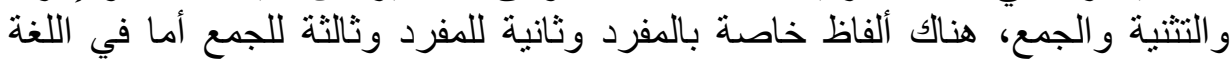

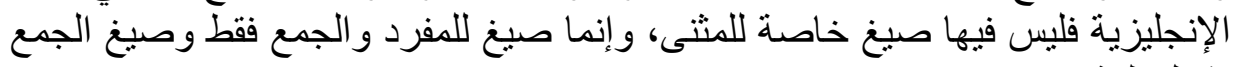
تشمل المثنى. 
ب - تقسيم الضمير في العربية محدود بينما تقسيمه في الإنجليزية متعددة، لأن فيها

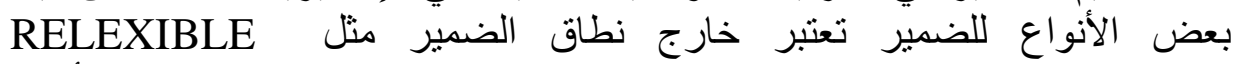
PRONOUN YOUR SELF OUR SELF التوكيد ( المعنوي).

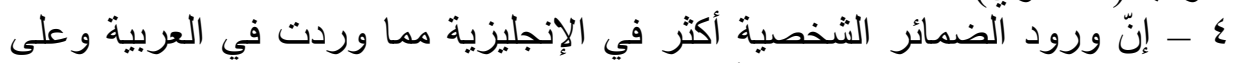

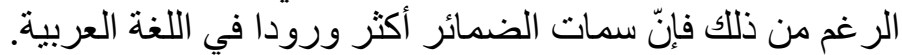

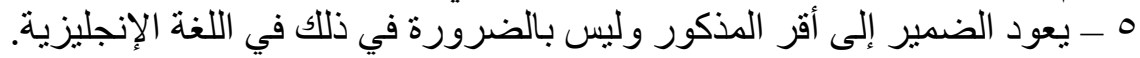

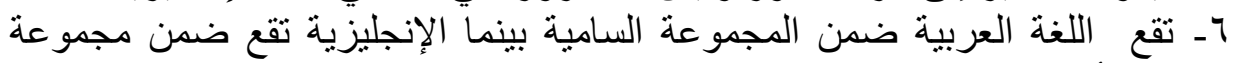
الهندية الأوروبية. النتائج:

في نهاية المطاف من هذا العمل فإنّ ظاهرة الضمير في اللغتين العربية والإنجليزية

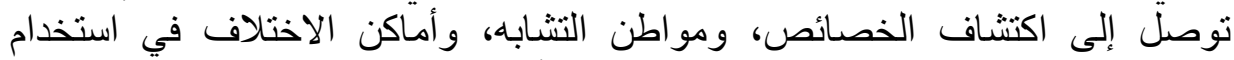

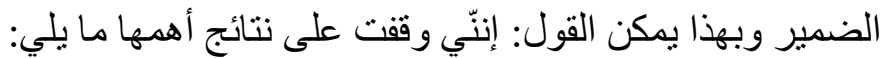

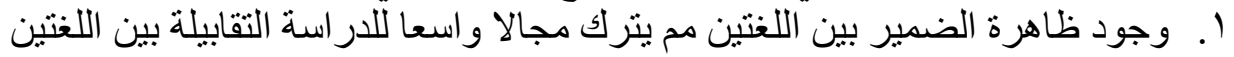
لبذه الظاهرة. r. إمكانية الجمع بين الضمائر و أسماء الإشارة و الموصول الفئة عند اللغتين.

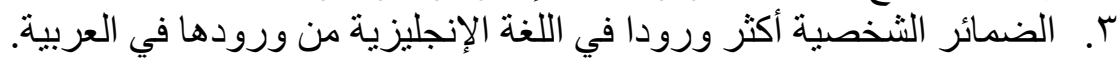

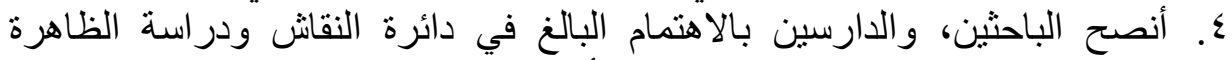

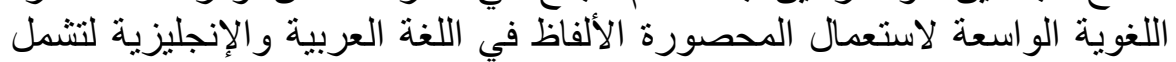

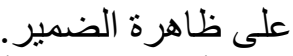
أخير ا : أحمد الله الذي أعانني على إنجاز هذا العمل المتواضع. 


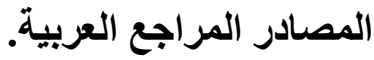

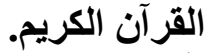

أبو بكر محمد بن السرب، الأصول في النحو، تا تل عبد الحسين الفتلي، مؤسسة الرسالة،

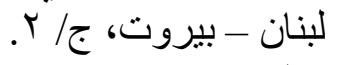

أبو حيان محمد بن يوسف بن بن علي بن يوسف بن حيان، البحر المحيط في التفسير،

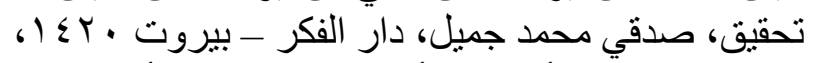

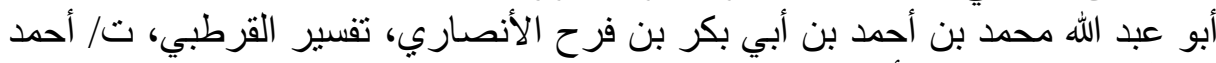

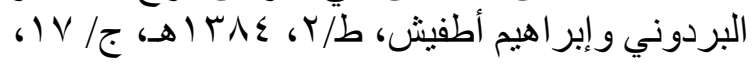

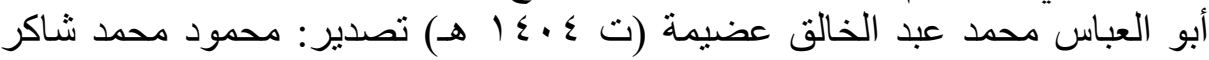

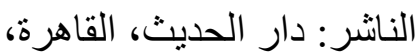

أبو العباس، شهاب الدين، أحمد بن يوسف ، الدارة الدر الدصون في الكتاب المكنون ت/

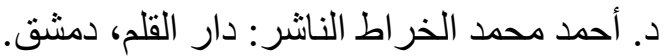
أبو الفتح عثمان بن جني الموصلي، الخصائص، الهيئة الدصرية العامة للكتاب، ط/ ـ / ل

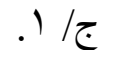
: أبو الفتح عثمان بن جني الموصلي سر صناعة الإعراب ا، النانشر: دار الكتب

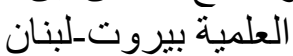

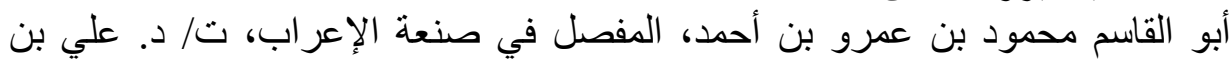

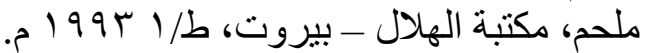
أبو القاسم محمود بن عمرو بن أحمد، الزمخشري الكثاف:، جار الله النانشر: دار، ،

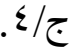
أبو محمد بدر الدين حسن بن عليّ المر ادي المصري، توضيح المقاصد و المسالك بشرح

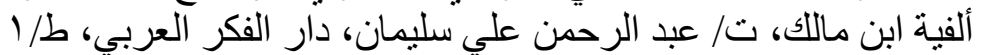

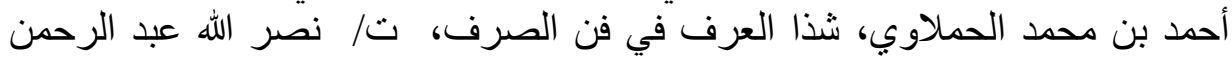
نصر الله النانشر: مكتبة الرشد الرياض.

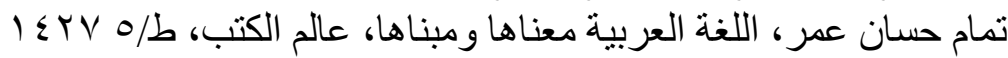

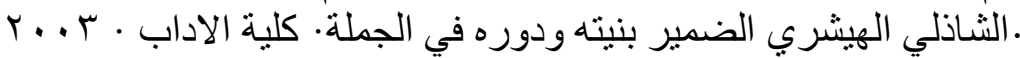

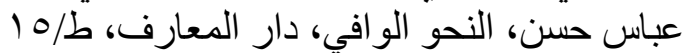

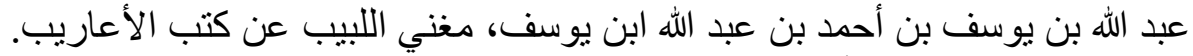

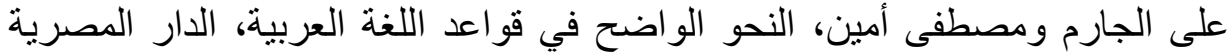

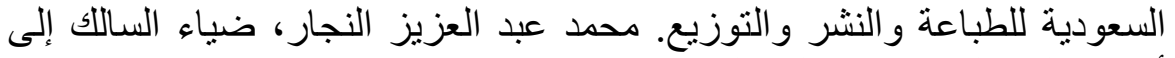

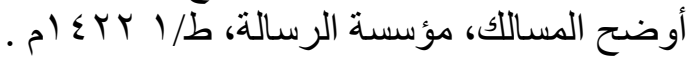




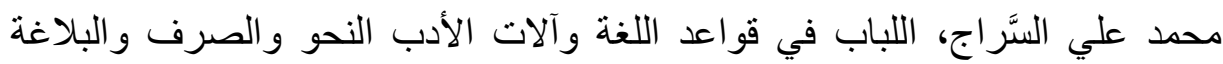

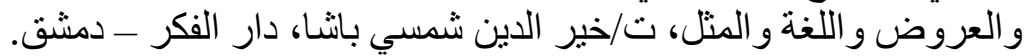

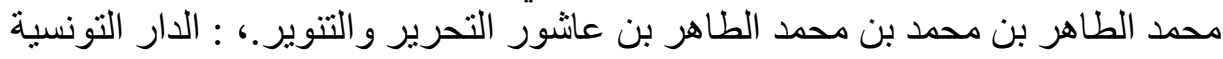

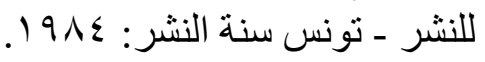

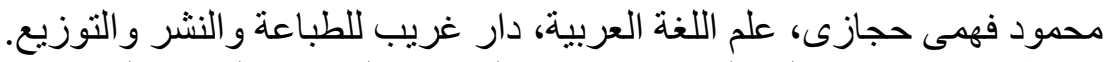

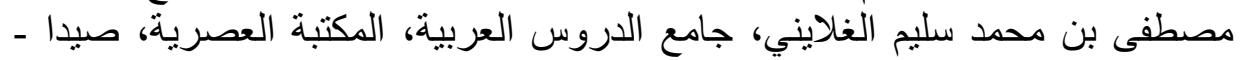

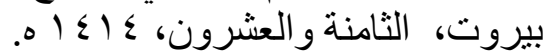
مجلة علمية.

جاسم بن علي، وزيدان علي جاسم، نظرية علم اللغة التقابلي في التراث العربي" مجلة

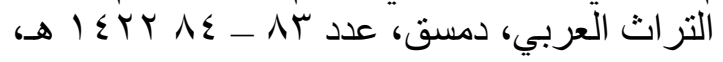
الرسائل العلمية.

عبد الحكيم بن عبد الله بن عبد الرحمن القاسم، عود الضمير وأثره في التفسير دراسة

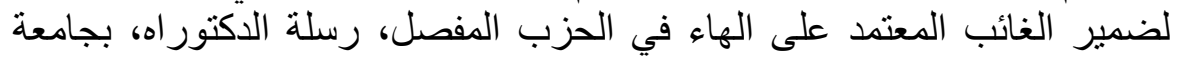
الملك سعود، كلية التربية.

المصادر الأجنبية.

Baecker, Diann L. "Uncovering the Rhetoric of the Syllabus: The Case of the Missing I." College

Bing, Janet. "Killing Us Softly: Ambiguous Markers of Power and Solidarity." Cultural

Performances: Proceedings of the Third Berkeley Women and Language Conference. April 1994..

John eastwood Ronald, Abasic English GRAMMAR,

J.C . Nesfield and F. T. Wood, MANUAL OF ENGLISH GRAMMAR \& COMPOSITION

Quick. R and Greenbaum . S University Grammar OF English (long man) London

$\mathrm{J}$. C NESFIED and F T WOOD, MANUAL OF ENGLISH GRAMMAR and COMPOSITION $4{ }^{\mathrm{TH}}$ EDITION

Gleason H . Linguistics and English Grammar C Holt, Reinhart and Winston New York London

D . N . S . BHAT . PRONOUNS . OXFORD UNIVERSITY PRESS. 
سبع عبد النافع أبانكندا الضمائر في اللغة العربية واللغة الإنجليزية .....

Sidney Greenbaum, personal pronouns in present day English, Cambridge universiry 1996 